\title{
Probabilistic Clustering of Extratropical Cyclones Using Regression Mixture Models
}

\author{
Scott J. Gaffney ${ }^{1}$, Andrew W. Robertson ${ }^{2 *}$ Padhraic Smyth ${ }^{3}$, \\ Suzana J. Camargo ${ }^{2}$ and Michael Ghil ${ }^{4,5}$ \\ ${ }^{1}$ Data Mining Research, Yahoo! Inc., \\ Sunnyvale, CA, USA \\ ${ }^{2}$ International Research Institute for Climate and Society (IRI), \\ The Earth Institute at Columbia University, Palisades, NY, USA \\ ${ }^{3}$ Department of Computer Science, \\ University of California, Irvine, CA, USA \\ ${ }^{4}$ Department of Atmospheric and Oceanic Sciences and IGPP, \\ University of California, Los Angeles, CA \\ ${ }^{5}$ Additional affiliation: Département Terre-Atmosphère-Océan \\ and Laboratoire de Météorologie Dynamique (CNRS and IPSL), \\ Ecole Normale Supérieure, Paris, France.
}

December 22, 2006

Climate Dynamics, sub judice

\footnotetext{
${ }^{*}$ Corresponding author: Dr. Andrew W. Robertson, IRI - Monell 230, 61 Route 9W, Palisades, NY 10964. Phone: +1 845680 4491, Fax: +1 845680 4865, E-mail: awr@iri.columbia.edu.
} 


\begin{abstract}
A probabilistic clustering technique is developed for classification of wintertime extratropical cyclone (ETC) tracks over the North Atlantic. We use a regression mixture model to describe the longitude-time and latitude-time propagation of the ETCs. A simple tracking algorithm is applied to 6-hourly mean sea-level pressure fields to obtain the tracks from either a general circulation model (GCM) or a reanalysis data set. Quadratic curves are found to provide the best description of the data.

We select a three-cluster classification for both data sets, based on a mix of objective and subjective criteria. The track orientations in each of the clusters are broadly similar for the GCM and reanalyzed data; they are characterized by predominantly south-to-north (S-N), west-to-east (W-E), and southwest-to-northeast (SW-NE) tracking cyclones, respectively. The reanalysis cyclone tracks, however, are found to be much more tightly clustered geographically than those of the GCM. For the reanalysis data, a link is found between the occurrence of cyclones belonging to different clusters of trajectory-shape, and the phase of the North Atlantic Oscillation (NAO). The positive phase of the NAO is associated with the SW-NE oriented cluster, whose tracks are relatively straight and smooth (with cyclones that are typically faster, more intense,
\end{abstract}


and of longer duration). The negative NAO phase is associated with more-erratic W-E tracks, with typically weaker and slower-moving cyclones. The $\mathrm{S}-\mathrm{N}$ cluster is accompanied by a more transient geopotential trough over the western North Atlantic. No clear associations are found in the case of the GCM composites.

The GCM is able to capture cyclone tracks of quite realistic orientation, as well as subtle associated features of cyclone intensity, speed and lifetimes. The clustering clearly highlights, though, the presence of serious systematic errors in the GCM's simulation of ETC behavior. 


\section{Introduction}

\section{a. Background and motivation}

Wintertime extratropical cyclones (ETCs) are responsible for severe-weather events with high winds and/or flooding over North America and western Europe; they caused the second largest insurance loss due to weather (after hurricanes) during the period 1990-98 (Saunders 1999). On the other hand they are also the primary source of wintertime precipitation and total water resources for much of the western United States.

ETCs play a special role as intermediaries between large-scale climate dynamics and local impacts: they are crucial dynamical ingredients of the atmospheric circulation, while at the same time directly impacting local weather. ETCs constitute an important nexus between the potentially predictable large-scale components of climate, such as certain hemispheric or sectorial atmospheric teleconnection patterns associated with internal climate variability (Lau 1988; Robertson and Metz 1990) or with global warming (Fyfe 2003), on the one hand, and societally important weather events (MunichRe 2002), on the other. A better understanding of the behavior of ETCs in the context of climate variability and change could have important societal implications.

ETCs have localized coherent spatial structures that generally propagate toward the east and 
go through a well-defined lifecycle (Simmons and Hoskins 1978). Their population is thus most naturally described as a set of moving objects that follow various tracks and have differing individual lifecycle characteristics; this corresponds to a Lagrangian description in fluid dynamicalterminology. By contrast, most data analysis in the atmospheric sciences has been based on calculating Eulerian statistics on spatially fixed grids, often using principal component analysis (Preisendorfer 1988; von Storch and Zwiers 1999) to derive the leading patterns of spatio-temporal variability. These methods are poorly suited to cyclone trajectories, which are inherently localized in space in any given time-frame.

The analysis of large sets of ETC trajectories, whether from multi-decadal observed data sets or from potentially much longer general circulation model (GCM) simulations requires a different approach. Cluster analysis provides a natural way to analyze sets of trajectories and their relationships with the larger-scale atmospheric circulation, by decomposing large sets of trajectories into subgroups with homogeneous spatio-temporal characteristics.

In this paper, we use curve-based mixture modeling techniques to perform probabilistic clustering of ETC trajectories in latitude-longitude space. Curve-based mixture modeling can be viewed as a particular clustering technique within the broader statistical framework of functional data analysis (Ramsay and Silverman 1997, 2002). An identification and tracking methodology is devel- 
oped to produce cyclone trajectories; these trajectories are then clustered using a novel probabilistic technique based on mixtures of regression models.

We develop and test the tracking and clustering methodology using a 15-winter GCM-generated mean sea level pressure (MSLP) data set. We then apply the methodology to a 44-year set of reanalysis data. Since the latter is a data assimilation of both meteorological observations and 6-hourly model forecasts, there is no guarantee of temporally smooth behavior.

\section{b. Related work}

Prior work on cyclone tracking has focused specifically on methods for automated identification and tracking of cyclones, usually from sea-level pressure data. Identification methods range from the relatively simple approach of finding minima in the surface pressure field (Le Treut and Kalnay 1990; König et al. 1993; Terry and Atlas 1996), or in the 5-point Laplacian thereof, to more complex approaches such as the use of image processing and computer vision techniques (Hodges 1994; Mesrobian et al. 1995; Hodges 1998); the latter approaches often involve other atmospheric fields such as vorticity (Hoskins and Hodges 2002). These algorithms are usually then coupled with a tracking scheme to produce a final set of trajectories. Methods proposed for tracking so far include a number of different schemes: nearest-neighbor search (Blender et al. 1997; König et al. 
1993), numerical prediction schemes with cost-minimizing optimizations (Murray and Simmonds

1991), and feature tracking methods from image analysis that are also based on a cost minimiza-

tion framework (Hodges 1994, 1995). Mailier et al. (2006) combined the tracking algorithms of

Hoskins and Hodges (2002) with a Poisson-based modeling approach to analyze clustering in time

of extratropical cyclone tracks in the Northern Hemisphere.

Blender et al. (1997) introduced the idea of using the $K$-means clustering algorithm to cluster ETC trajectories of fixed length. The $K$-means algorithm iteratively searches for compact clusters of multidimensional points in $d$-dimensional Euclidean space (Hartigan and Wong 1978); this algorithm minimizes within-cluster variance for a given number $K$ of clusters. To apply the $K$ means algorithm to cyclone trajectory data, one must first convert the variable-length trajectories into fixed-dimensional vectors. To do this, Blender et al. (1997) constrained each of their storm trajectories to be exactly 3 days in length and then concatenated each of the latitude and longitude measurements to form the vectors on which the $K$-means algorithm operates. Elsner et al. (2000) and Elsner (2003) used the $K$-means algorithm to cluster tropical cyclone trajectories based on the latitude and longitude locations of storms when they reach specific intensities.

This type of vector-based clustering has limitations when applied directly to trajectories. For example, the conversion of the time and space measurements into a fixed-dimensional vector-space 
loses spatio-temporal smoothness information related to the underlying dynamics of the ETC process, whereas the mixture-based approach uses trajectory models (for each mixture component) that are smooth as a function of time. Furthermore, the vector-based approach artificially constrains the trajectories to have fixed lengths. The regression-based clustering used in this paper has been shown to provide systematically better fit and more accurate predictions when used to cluster variable-length trajectory data, compared to vector-based clustering (Gaffney and Smyth 1999; Gaffney 2004). Allowing for tracks of varying lengths may be of particular significance; for example, Simmons and Hoskins (1978) identified a lifecycle of about 10 days, much longer than that assumed by Blender et al. (1997). The approach we propose in this paper for ETC clustering, namely mixtures of regression models, directly incorporates spatio-temporal smoothness in the trajectories in the modeling process, and accommodates cyclone trajectories of different lengths.

Hierarchical clustering could also be used in this context by defining a distance between pairs of trajectories. For example, dynamic time-warping techniques could be used to define a transformation distance between any curve and another (Wang and Gasser 1997). Both hierarchical clustering and $K$-means clustering, however, do not allow for a consistent and systematic approach to problems such as assessing the predictive performance of a cluster model, model selection, or handling missing data. In contrast, the probabilistic, regression-based approach to clustering provides a sta- 
tistical basis to systematically address these issues (Fraley and Raftery 1998, 2002; Smyth 2000;

McLachlan and Peel 2000).

\section{c. Purpose and outline}

Our purpose in this paper is threefold: (i) to develop further the methodology for ETC tracking and classification; (ii) to apply this methodology to North Atlantic ETCs that impact European climate; and (iii) to evaluate the performance of a typical GCM in simulating the observed ETC clusters.

The paper is organized as follows. Section 2 presents our cyclone identification and tracking methodology and describes the data sets used in this paper. Section 3 introduces a new curve-based methodology for ETC clustering in two parts: (i) a brief introduction to finite mixture models; and (ii) their extension to regression mixture models and the integration of cyclones into this framework. Section 4 presents the clustering results for the GCM data, while the corresponding results for the reanalysis data set appear in Section 5. The ETC clusters are related to the large-scale meteorological fields in Section 6, followed by concluding remarks in Section 7. 


\section{Data and Tracking Methodology}

a. Data

The GCM data set used for this work was generated by the National Center for Atmospheric Research (NCAR) Community Climate Model (CCM3) (Hack et al. 1998). The model is discretized in spherical harmonics at a T42 resolution, and forced with observed sea surface temperatures specified at the lower boundary over the 1980/81-1994/95 period. For the tracking, we used 6hourly MSLP fields on the model's approximate $2.8^{\circ} \times 2.8^{\circ}$ Gaussian grid for extended 6-month winter months (1 November to 30 April) from 1980 to 1995 ; in each winter there are thus 181 days. In this paper we focus on North Atlantic ETCs over the area $\left(30^{\circ} \mathrm{N}-80^{\circ} \mathrm{N}, 80^{\circ} \mathrm{W}-30^{\circ} \mathrm{E}\right)$ shown in Fig. 1.

[Figure 1 near here, please.]

The reanalysis data set used in Section 5 is the National Centers for Environmental Prediction (NCEP)-NCAR data assimilation of historical observations using a state-of-the-art analysis/forecast system (Kalnay et al. 1996). We use 6-hourly MSLP on a regular latitude-longitude grid of $2.5^{\circ} \times 2.5^{\circ}$, over the same North Atlantic domain as for the GCM, but for the 44 extended winter seasons 1958/59-2001/02. 
To cluster ETC trajectories we must first identify and track them from the MSLP frames. Our identification and tracking scheme is based on methods already used in this context (Blender et al. 1997; König et al. 1993) and requires relatively few parameters to implement. The tracking algorithm we use is quite simple and not intended as a general-purpose tracking algorithmnonetheless, we found that it produced reliable results on the data sets used in this paper. We only give a short description below; the full details can be found in Gaffney (2004).

\section{b. Identifying and tracking cyclones}

Cyclones are characterized as well-defined surface-pressure minima and their trajectories have lengths of a few thousand kilometers. We begin with a minimum-finding procedure to locate candidate centers of cyclones within each field. In order to distinguish these minima more easily from larger-scale low-pressure areas, the gridded data were spectrally filtered in space at each time so as to remove the largest planetary-wave scales; these scales were defined as the first five global spherical harmonics (Hoskins and Hodges 2002; Anderson et al. 2003). Using bicubic interpolation, a cyclone center that may be off-grid is then obtained.

Spurious minima can arise using this procedure, usually in one of two situations: (1) in highpressure regions not associated with cyclonic activity; and (2) on the outskirts of a single cyclonic 
system with an already located central minimum. These spurious minima are automatically identified and removed from further analysis, by thresholding the MSLP data at a particular pressure level to form individual low-pressure regions within the data. This thresholding results in contiguous local pixel regions, where each local region corresponds to the estimated spatial extent of a single candidate cyclone at a specific time. We then reject minima that are located outside the low-pressure regions and only keep the deepest minimum within each individual local region. The threshold was defined via trial-and-error to be $-17 \mathrm{hPa}$, a subjective choice that produced intuitive results - the set of tracks obtained are relatively insensitive to small variations in the value of the threshold (Gaffney 2004). The same threshold value is used for all times $t$.

Once the valid MSLP minima have been detected at successive 6-hour time intervals, they are linked together over time to form cyclone tracks. Each valid minimum at time $t$ is linked with the closest minimum that was detected within a small neighborhood in the MSLP field from $t-6$ hours. This search is carried out within $\pm 7^{\circ}$ long. and $\pm 5^{\circ}$ lat. These bounds correspond to a maximum velocity of approximately $92 \mathrm{~km} / \mathrm{h}$ in longitude and latitude, at $45^{\circ} \mathrm{N}$; in practice such velocities are hardly, if ever, reached. In the second step, we eliminate tracks shorter than 2.5 days. This removes many short and noisy tracks that correspond to local small-scale weather disturbances not usually considered to be synoptic cyclones. 
Application of this identification and tracking procedure to the MSLP data from CCM3 produced 614 cyclones of different durations, each with a minimum of 10 time-steps (i.e., at least 2.5 days long). Figure 1 shows a sample of the resulting cyclone tracks, with starting positions indicated by circles. Figure 2 contains three summary histograms describing the statistical characteristics of the entire set of trajectories. The cyclone tracks have typical durations of 2.5-4 days, typical velocities of 30-60 km/hr (i.e. $8-16 \mathrm{~m} / \mathrm{s}$ ), and reach typical maximum intensities, defined as the minimum MSLP reached, of -30 to $-50 \mathrm{mb}$. These values are of the same order as the statistics derived from tracking observed cyclones in other studies (Hoskins and Hodges 2002). We use this set of trajectories as input to our clustering algorithm in what follows.

[Figure 2 near here, please.] 


\section{Clustering methodology}

a. Finite mixture models and model-based clustering

In the standard mixture model framework, we model the probability density function (PDF) for a $d$-dimensional vector $\mathbf{x}$, as a function of model parameters $\phi$, by the mixture density

$$
p(\mathbf{x} \mid \phi)=\sum_{k}^{K} \alpha_{k} p_{k}\left(\mathbf{x} \mid \theta_{k}\right),
$$

in which $\phi=\left\{\alpha_{1}, \ldots, \alpha_{K} ; \theta_{1}, \ldots, \theta_{K}\right\}, \alpha_{k}$ is the $k$-th component weight, and $p_{k}$ is the $k$-th component density with parameter vector $\theta_{k}$; for example, $K$ Gaussian densities each with a $d$ dimensional mean vector and a $d \times d$ covariance matrix. The mixture weights $\alpha_{k}$ sum to one and are nonnegative. In this manner a finite mixture model is a PDF composed of a weighted average of component density functions (McLachlan and Peel 2000).

The mixture model framework can be used for data clustering as follows. A data set of $n$ vectors $\left\{\mathbf{x}_{1}, \ldots, \mathbf{x}_{n}\right\}$ is observed, and is assumed to be a random sample from the underlying mixture model. Each data vector $\mathbf{x}_{i}$ is generated by one of the $K$ components, but the identity of the generating component is not observed. The parameters for each density component $p_{k}\left(\mathbf{x} \mid \theta_{k}\right)$, as well as 
the corresponding weights $\alpha_{k}$, can be estimated from the data using the Expectation-Maximization (EM) algorithm, a widely used technique for maximum-likelihood parameter estimation with mixture models (Dempster et al. 1977a; McLachlan and Krishnan 1997). From a clustering viewpoint, the estimated component models, $p_{k}\left(\mathbf{x} \mid \theta_{k}\right), 1 \leq k \leq K$, are interpreted as $K$ clusters, where each cluster is defined by a PDF in the $d$-dimensional input space $\mathbf{x}$.

Furthermore, using Bayes rule and Eqn. (1), the probability that $\mathbf{x}$ was generated by the $k$ th cluster (or component) can be calculated (Eqn. (A2 in Appendix A). These membership probabilities reflect the a posteriori uncertainty (given the data and the model) about which cluster each data vector $\mathbf{x}_{i}$ originated from. A "hard" clustering of the original data $\left\{\mathbf{x}_{1}, \ldots, \mathbf{x}_{n}\right\}$ can be inferred by assigning each vector $\mathbf{x}_{i}$ to the cluster $f_{k}$ with the highest membership probability, i.e., the cluster from which it was most likely generated. Finite mixture models have been widely used for clustering data in this manner in a variety of application areas (e.g., McLachlan and Basford 1988) including atmospheric sciences (e.g., Smyth et al. 1999; Hannachi and O'Neill 2001; Vrac et al. 2005). 


\section{b. Cyclone regression mixture models}

Regression mixture models are a direct extension of the vector mixture models described above.

For illustration, consider a hypothetical trajectory $\mathbf{z}_{i}$ with $n_{i}=4$ measurements; the longitude and latitude measurements are in the first and second column and their initial values have been subtracted, while $\mathbf{t}_{i}$ is the elapsed time from initiation of tracking:

$$
\mathbf{z}_{i}=\left[\begin{array}{cc}
0 & 0 \\
1 & 0.2 \\
2.5 & 0.4 \\
3.3 & 0.7
\end{array}\right], \quad \mathbf{t}_{i}=\left[\begin{array}{l}
0 \\
1 \\
2 \\
3
\end{array}\right] .
$$

Note that this example represents a cyclone moving mostly in a zonal direction. The methodology below is developed for the case where each trajectory $\mathbf{z}_{i}$ is represented by two-dimensional lat-lon measurements over time. However, the framework can also handle additional dimensionality in $\mathbf{z}_{i}$, e.g., 3-dimensional positional vectors could be used to offset distortions introduced by lat-lon projections, and additional non-positional attributes such as intensity as a function of time could also be added.

We model longitudinal position with a polynomial regression model of order $p$ in which time 
$\mathbf{t}_{i}$ is the independent variable, e.g., $z=\beta_{p} t^{p}+\beta_{p-1} t^{p-1}+\ldots+\beta_{1} t+\beta_{0}$, and likewise for latitude.

Both regression equations can be defined succinctly in terms of the matrix $\mathbf{T}_{i}$ :

$$
\mathbf{z}_{i}=\mathbf{T}_{i} \boldsymbol{\beta}+\epsilon_{i}, \quad \epsilon_{i} \sim N(\mathbf{0}, \Sigma) .
$$

Here $\mathbf{T}_{i}$ is the standard $n_{i} \times(p+1)$ Vandermonde regression matrix associated with the vector $\mathbf{t}_{i} ; \boldsymbol{\beta}$ is a $(p+1) \times 2$ matrix of regression coefficients, which contains the longitude coefficients in the first column and the latitude coefficients in the second column; and $\epsilon_{i}$ is an $n_{i} \times 2$ matrix of multivariate normal errors, with a zero mean and a $2 \times 2$ covariance matrix $\Sigma$. Assuming that the noise term $\epsilon$ is normal (Gaussian) is equivalent in effect to using a least-squares loss function in regression fitting. Alternative noise models could also be considered—we used the normal assumption here since it is the most straightforward computationally and is a common choice for additive noise in regression models.

The Vandermonde regression matrix $\mathbf{T}_{i}$ consists of $(p+1)$ columns of $\mathbf{t}_{i}$ so that the components of $\mathbf{t}_{i}$ in the $m$-th column are taken to the power of $m$ for $0 \leq m \leq p$. For example, if $p=2$ and 
$\mathbf{t}_{i}=(0,1,2,3)^{\prime}$, where ()$^{\prime}$ denotes the transpose, then

$$
\mathbf{T}_{i}=\left[\begin{array}{lll}
0 & 0 & 0 \\
1 & 1 & 1 \\
1 & 2 & 4 \\
1 & 3 & 9
\end{array}\right] .
$$

The covariance matrix $\Sigma$ contains three distinct elements: the noise variances $\sigma_{1}^{2}$ and $\sigma_{2}^{2}$ for each longitude and latitude measurement, respectively, and the cross-covariance $\sigma_{12}=\sigma_{21}$ between any two longitude and latitude measurements. For simplicity, we make the assumption that $\Sigma=$ $\operatorname{diag}\left(\sigma_{1}^{2}, \sigma_{2}^{2}\right)$, so that latitude and longitude measurement noise terms are treated as conditionally independent given the model. While a non-diagonal covariance matrix would allow for modeling of dependence between the latitude and longitude measurement noise terms, this level of detailed modeling did not appear necessary for the purposes of clustering and, thus, we used a diagonal covariance assumption as a simpler alternative.

The conditional density for the $i$-th cyclone is thus defined as

$$
p\left(\mathbf{z}_{i} \mid \mathbf{t}_{i}, \theta\right)=f\left(\mathbf{z}_{i} \mid \mathbf{T}_{i} \boldsymbol{\beta}, \Sigma\right)
$$




$$
=(2 \pi)^{-n_{i}}|\Sigma|^{-n_{i} / 2} \exp \left\{-\frac{1}{2} \operatorname{tr}\left[\left(\mathbf{z}_{i}-\mathbf{T}_{i} \boldsymbol{\beta}\right) \Sigma^{-1}\left(\mathbf{z}_{i}-\mathbf{T}_{i} \boldsymbol{\beta}\right)^{\prime}\right]\right\}
$$

where the parameter set $\theta=\{\boldsymbol{\beta}, \Sigma\}$ contains the regression coefficients $\boldsymbol{\beta}$ and the noise covariance matrix $\Sigma$.

We can derive regression mixtures for the cyclones by substitution of the unconditional multivariate density components $p_{k}\left(\mathbf{x} \mid \theta_{k}\right)$ in Eqn. (1) with the conditional regression density components $p_{k}\left(\mathbf{z} \mid \mathbf{t}, \theta_{k}\right)$, defined in Eqn. (3) above. This results in the following regression mixture model for ETC trajectories:

$$
p\left(\mathbf{z}_{i} \mid \mathbf{t}_{i}, \phi\right)=\sum_{k}^{K} \alpha_{k} p_{k}\left(\mathbf{z}_{i} \mid \mathbf{t}_{i}, \theta_{k}\right)=\sum_{k}^{K} \alpha_{k} f_{k}\left(\mathbf{z}_{i} \mid \mathbf{T}_{i} \boldsymbol{\beta}_{k}, \Sigma_{k}\right) .
$$

Note that in this model each ETC is assumed to be generated by one of $K$ different regression models, and each model has its own "shape" parameters $\theta_{k}=\left\{\boldsymbol{\beta}_{k}, \Sigma_{k}\right\}$. The technique is quite general and can be adapted to many types of regression models including linear (DeSarbo and Cron 1988), binomial probit (Lwin and Martin 1989), kernel (Gaffney and Smyth 1999), and random effects (Lenk and DeSarbo 2000; Gaffney and Smyth 2003) models. 


\section{c. Clustering trajectories via regression mixture models}

If we let $\boldsymbol{Z}=\left\{\mathbf{z}_{1}, \ldots, \mathbf{z}_{n}\right\}$ be the complete set of $n$ cyclone trajectories and $\boldsymbol{T}=\left\{\mathbf{t}_{1}, \ldots, \mathbf{t}_{n}\right\}$

be the set of associated measurement times, then the full probability density of $\boldsymbol{Z}$ given $\boldsymbol{T}$, also known as the conditional likelihood, is

$$
p(\boldsymbol{Z} \mid \boldsymbol{T}, \phi)=\prod_{i}^{n} \sum_{k}^{K} \alpha_{k} f_{k}\left(\mathbf{z}_{i} \mid \mathbf{T}_{i} \boldsymbol{\beta}_{k}, \Sigma_{k}\right) .
$$

The model can handle trajectories of variable length in a natural fashion, since the likelihood equation (5) above does not require the number of data points in each ETC $\mathbf{z}_{i}$ to be the same; there is no need, therefore, to truncate trajectories to any predefined common length. This probabilistic model can also be extended in a straightforward manner to incorporate additional information into the clustering (such as storm intensity as a function of time) by augmenting the $Z$ variable with additional dimensions.

The product form in Eqn. (5) follows from assuming conditional independence of the $\mathbf{z}_{i}$ 's, given both the $\mathbf{t}_{i}$ 's and the mixture model, that is assuming ETCs do not influence each other. Strictly speaking this is not necessarily true, since multiple ETCs could be present at the same time and there can be "clustering in time" effects for ETCs (Mailier et al. 2006). However, for the 
purposes of clustering trajectories based on their shape, the conditional independence assumption in the likelihood above is quite reasonable. The cluster membership of a particular ETC is likely to primarily depend on how similar the shape of the trajectory is to each of the clusters, and only much more weakly on information from ETCs that come before or after it in time.

Clustering is performed by (a) learning the parameters of all $K$ models given data; and then (b) inferring for each ETC which of the $K$ clusters it belongs to. Following Blender et al. (1997), each cyclone trajectory is referred to the origin in both space and time, so that each $\left(\mathbf{z}_{i}, \mathbf{t}_{i}\right)$ begins at the relative latitude-longitude position $(0,0)$ and at a time $t=0$. Clustering is thus performed using only the shape of the trajectory, and initial starting positions are eliminated as a source of variation.

An EM algorithm for learning the component regression models and component weights for this conditional mixture can be defined in a similar manner to the EM algorithm for standard (unconditional) mixtures (McLachlan and Peel 2000; DeSarbo and Cron 1988; Gaffney and Smyth 1999). The maximization (M) step consists of solving a weighted least-squares regression problem in which the weights are the membership probabilities calculated in the expectation (E) step. Complete details on implementing the EM algorithm are described by Gaffney and Smyth (1999) and Gaffney (2004), and an outline is provided in Appendix A. 
A graphical example of using EM to estimate the parameters of regression mixtures from simulated curve data is shown in Fig. 3; a single space dimension is used here for illustration purposes. Four curves were generated from each of three underlying quadratic polynomials, for a total of 12 curves (Fig. 3a); i.e., four samples (different line types in Fig. 3a) were drawn from each of the clusters. Note that the cluster "labels," shown here using the x-es, circles, and squares in Fig. 3a, were not given to the algorithm. Figure $3 \mathrm{~b}$ shows the initial, randomly chosen starting trajectory of the algorithm for each of the three regression models. The EM algorithm converges in 4 iterations and the final clustering is shown in Fig. 3c, along with the classification of each curve resulting from the clustering (shown by the x-es, circles, and squares respectively); the latter is $100 \%$ accurate in this simple example (compare with the same symbols in Fig. 3a). The underlying true polynomials that generated the data are the dotted lines in Fig. 3c. The regression mixture methodology recovers the true cluster structure from the data, even though it is not visually apparent at all that the top two clusters in Fig. 3a can be separated.

[Figure 3 near here, please.]

A quadratic polynomial is also used in our component regression models for the ETC tracks. This choice was based on visual inspection of fitted-versus-actual trajectory data (see Fig. 4) as well as on a quantitative cross-validation analysis. In the latter (not shown), we fitted regression mixture 
models with different orders of polynomial to randomly selected training sets of trajectories, and then computed the log-probability of unseen "test" trajectories under each model. This calculation was repeated $C=10$ times over multiple training-test partitions of the data to generate average out-of-sample log-probability (or log-likelihood) scores (Smyth 2000; Smyth et al. 1999).

[Figure 4 near here, please.]

The $\log -p$ score for a set of trajectories is defined as the log of Eqn. (5) for a model with parameters estimated from a different (training) data set. The higher (i.e., more positive) the outof-sample $\log$ - $p$ score the better a model is in terms of capturing the structure of the true probability density generating the data (Bernardo and Smith 1994; Gneiting and Raftery 2004).

\section{Clustering of cyclones in GCM simulations}

This section describes the results obtained from applying the clustering methodology of Sect. $3 \mathrm{~b}$ to the 15 extended winter seasons of GCM cyclone trajectories; see Sect. 2b. An important question is the selection of the number of cyclone clusters. Figure 5 shows the cluster-specific mean curves of each regression mixture model fitted to the cyclone data for $K=2,3,4$, and 6 clusters. Each graph plots the cluster mean in relative latitude-longitude space, using trajectories referred to the origin. Blender et al. (1997) set the number of clusters to three based on various meteorological 
considerations. In a similar manner, the three-cluster model in Fig. 5 provides a large-scale description of the North Atlantic cyclones. As the number of clusters is increased, the individual clusters tend to split into smaller refinements of the simpler cluster models, as seen in Fig. 5 for $K=4$ and $K=6$ (bottom panels).

[Figure 5 near here, please.]

Objective goodness-of-fit measures can also be defined to help in determining the "best" number of clusters. We used both the cross-validated (or out-of-sample) log-likelihood and predicted sum of squared errors (SSE) to investigate whether the data set itself could objectively identify the number of clusters. Figure 6 shows both scores as a function of the number of clusters $K$. The SSE is calculated by predicting each point in the last half of the curves given the first half; the predictions were made sequentially so that the last point was predicted given the entire rest of the curve. Since the predicted curve in each cluster is just the cluster mean (when cluster membership is close to 1), this is close to the spread of each cluster. Both the measures of fit behave in a nearmonotonic manner as $K$ is increased, so that it is not possible to objectively identify an optimal value using these scores alone. Still, from the MSE plot in particular, beyond a range of about $K=3$ to $K=7$ clusters, there are diminishing returns from further increasing $K$.

[Figure 6 near here, please.] 
Even though the purely objective measures above provide limited guidance in choosing $K$, we will demonstrate in the rest of the paper that $K=3$ provides a meaningful "coarse-grained" description of ETC behavior in both the GCM and reanalysis data; in particular, this number of clusters provides the clearest comparison between the two data sets. Similar plots were also obtained using the reanalysis data (see Sect. 5 below).

The three clusters are named "south-to-north" (S-N), "southwest-to-northeast" (SW-NE), and "west-to-east" (W-E); they are labeled according to their latitude-longitude orientation on the page: V (“vertical”), D (“diagonal”), and H (“horizontal”) in Fig. 7, which shows a sample of 100 trajectories assigned to each cluster. The number of trajectories in each cluster is 220 for V, 215 for $\mathrm{D}$, and 179 for $\mathrm{H}$, out of a total of 614 cyclone tracks identified in the CCM3 simulation.

[Figure 7 near here, please.]

The characteristics of each cluster are given in Table 1. The velocity is based on the difference between two successive positions, 6 hours apart, along a given trajectory; the average acceleration is then the difference between two successive velocities, while the curvature of an ETC is based on the instantaneous curvature values obtained from the quadratic polynomial fitted at each point multiplied by 1000. Averages of these quantities are then computed over each cluster. The noisiness of a cyclone track estimates the degree of "erratic" departure from a smooth path, which is calculated 
by the standard deviation of instantaneous curvature along the trajectory (also multiplied by 1000).

[Table 1 near here, please.]

The V-cluster consists of relatively short, south-to-north oriented cyclones with large curvature and noisiness. The cyclones in this cluster are fairly slow with many exhibiting relatively stationary behavior. The D-cluster consists of a large group of diagonally oriented cyclones that generally cross the Atlantic travelling from south-west to north-east. These cyclones have the largest average velocity $(59 \mathrm{~km} / \mathrm{h})$, intensity ( $-40 \mathrm{mb})$, duration (4.1 days), and the smallest noisiness (7.8), as compared to those in the other two clusters. The H-cluster consists of cyclones that move west to east, across the western coastlines of Europe. These cyclones are the least intense on average ( -34 $\mathrm{mb}$ ) overall, but have the largest acceleration values $\left(19 \mathrm{~km} / \mathrm{h}^{2}\right)$ and curvature (15); a large part of the curvature can be attributed to erratic behavior, as reflected by their large noisiness of 23 .

Figure 8 shows histograms of average speed, duration and maximum intensity, stratified by cluster. Cluster D contains the fastest cyclones in the overall set, with several having average speeds greater than $80 \mathrm{~km} / \mathrm{h}$. Cluster V contains the largest number of short-duration tracks, lasting 3 days or less, and only $6 \%$ of the cyclones in cluster $\mathrm{V}$ last longer than 5 days, as opposed to $11 \%$ and $18 \%$ for clusters $\mathrm{H}$ and $\mathrm{D}$, respectively.

[Figure 8 near here, please.] 


\section{Clustering of cyclones in reanalysis data}

In this section we apply our new clustering methodology to reanalyzed cyclone trajectories over the 44 extended winter seasons. These were tracked in the same manner as the GCM trajectories, using the same region of $30^{\circ} \mathrm{N}-80^{\circ} \mathrm{N}$ and $80^{\circ} \mathrm{W}-30^{\circ} \mathrm{E}$ as in the $\mathrm{GCM}$ analysis; the resulting set contains 1,915 ETC trajectories. This number is about 3 times larger than the number found in the

GCM data, consistent with the reanalysis data covering about 3 times as many seasons (44 versus

15). Cyclones are active on approximately $75 \%$ of the days in the 44 -winter data set. Summary histograms (not shown) of average velocity and maximum intensity are quite similar to those in Fig. 2 for the GCM data.

We again selected $K=3$ clusters for analysis, for ease of comparison with the GCM case. Plots of log-likelihood and SSE scores (not shown) exhibit similar features to those in Fig. 6 for the GCM case. There is thus no clear evidence that the appropriate value of $K$ should be any different, while selecting a common value of $K=3$ in both analyses allows for a straightforward comparison of ETC behavior in the two datasets. This choice was found in Sect. 6 to yield clusters related to distinct physical phenomena, including the North Atlantic Oscillation (NAO).

The trajectories were first referred to their initial positions, as for the GCM data, but not otherwise normalized. Figure 9 shows the tracks from each of the three clusters. 
[Figure 9 near here, please.]

As in the GCM data set (Fig. 7), the clusters show predominantly vertical (V), diagonal (D), and horizontal $(\mathrm{H})$ track orientations. The three reanalysis clusters are almost equally populated, with 680, 604 and 631 trajectories, respectively. The V-cluster has meridional, recurving tracks, as in CCM3, but is much more heavily concentrated over the western North Atlantic than in the GCM; it contains the largest number of members. The reanalysis D-cluster also forms a much narrower diagonal, SW-NE swath of tracks across the Atlantic. Indeed, the GCM's cyclones are generally too zonal in their spatial distribution, extending excessively into Europe. The H-cluster has predominantly eastward oriented tracks but its track distribution is more erratic than in the GCM.

Compared to the three clusters of Blender et al. (1997, Fig. 3 there), who used a higherresolution data set, based on operational analyses of the European Centre for Medium-Range Weather Forecasts (ECMWF) for 1990-94, our results do not include the "stationary" cyclones over Greenland and the Mediterranean. Our D-cluster can be equated with the "northeastward" cluster of these authors, and our H-cluster with their "zonal" one. The largest difference between the results of Blender and colleagues and those reported here is our heavily populated V-cluster, with most trajectories close to the coastline of North America. 
[Figure 10 near here, please.]

Figure 10 shows histograms of average speed, duration and maximum intensity for the reanalysis trajectories, stratified by cluster, with summary statistics given in Table 2; these display items are analogous to Fig. 8 and Table 1 for the GCM trajectories. The overall statistical results for the reanalysis are generally quite consistent with the GCM case (Table 2 vs. Table 1), while some discrepancies appear in the detailed distribution of the ETC tracks (Fig. 10 vs. Fig. 8). The D-cluster again contains markedly faster moving cyclones (mean velocity of $63 \mathrm{~km} / \mathrm{h}$ ), with the H-cluster containing the slowest (mean of $38 \mathrm{~km} / \mathrm{h}$ ). The durations are more similarly distributed between the clusters than in the GCM. The reanalysis H-cluster contains less intense cyclones, qualitatively similar to the GCM. The reanalysis accelerations are slightly larger in all three cases, and lifetimes are slightly shorter and differ little between the reanalysis clusters (3.5-3.6 days). In addition, the reanalysis data set shows relatively fewer D-cluster cyclones, but relatively more D and $\mathrm{H}$ tracks of short duration.

The largest differences between reanalysis and GCM-simulated cyclones are in the curvature and smoothness of the tracks. The reanalysis cyclones exhibit much smaller curvature and noisiness, across all three clusters. Our results thus indicate that the data assimilation scheme used in the NCEP-NCAR reanalysis produces fairly smooth ETC trajectories. As in the GCM case, the 
D-cluster cyclones in the reanalysis tend to have tracks that are straighter and less noisy.

\section{Meteorological Composites}

Storm-track activity associated with ETCs is typically examined in terms of Eulerian eddy statistics

(e.g., Blackmon et al. 1977; Hurrell et al. 2003), so that it is of interest to compare our Lagrangian track-based clusters with composites of sub-weekly variance for each cluster. A simple compositing approach follows naturally from the discrete nature of the clustering, which can be contrasted with the regression approach of Mailier et al. (2006). To construct the composites, a day is assigned to a cluster if a cyclone from that cluster is active on that day. If no cyclones are active, the day is assigned to a fourth, "quiescent" regime. For days with overlap, the regime corresponding to the cluster with the largest number of active cyclones on that day is chosen. In the case of a tie between two or more active clusters, the regime which was most recently selected corresponding to one of the "tied" clusters is chosen; this criterion can be thought of as a type of "inertial bias". In the GCM (reanalysis) data, overlap occurs on $15.5 \%$ (18.4\%) of days.

Composites of anomalous storm-track activity are plotted in Fig. 12 for each cluster, as anomalies in the high-pass filtered MSLP variance; these anomalies are defined with respect to the climatological averages of high-pass variance displayed in Fig. 11. A 7-point high-pass filter with a 
6.4-day cut-off was used to isolate the ETC variability (Trenberth 1986). The climatological storm tracks exhibit broadly similar geographical distributions in the reanalysis and GCM MSLP fields (Figs. 11a,b), with maxima near Newfoundland and Iceland.

[Figure 11 and 12 near here, please.]

The composites of anomalous variance tend to be consistent with the geographical track distributions of the respective clusters in Figs. 7 and 9. They show some broad similarities between the two data sets, but with some important differences. In general, the reanalysis composites are much more spatially coherent, which is consistent with the larger degree of geographical localization seen in the corresponding trajectory clusters. In both cases, the "Quiescent" cluster, which comprises days when no cyclones are active, shows decreased variance over much of the North Atlantic. The H-cluster also shows a decreased variance over most of the North Atlantic, with slight enhancement of variance off the coasts of western Europe. The V and D clusters differ the most between reanalysis and GCM, with the former showing very marked increases in cyclone activity in the regions expected from Fig. 9. The GCM's V and D clusters show enhanced variance near $60^{\circ} \mathrm{N}$, consistent with Fig. 7. In summary, while the clustering is based soley on trajectory shape, the resulting clusters exhibit distictively differing geographical distributions of high-pass MSLP variance, and this is especially clear in the reanalysis data. 
One of the motivations for clustering cyclone trajectories is to relate differing cyclone types to the larger-scale background flow. To this end, we have low-pass filtered 700-hPa geopotential heights in the reanalysis data, with a 10-day cut-off, and composited the resulting fields for each cluster. The low frequencies are selected so as to focus on the component of the circulation that is not directly associated with the cyclones themselves. Maps of the four composites, based on departures from the grand mean of the 44 winters, are plotted in Fig. 13. All four composites are characterized by geopotential height anomalies that closely mirror the distributions of ETC tracks and sub-weekly MSLP variance. The quiescent composite shows a ridge over the climatological position of the storm track, consistent with reduced cyclone activity, and a weak trough west of Greenland. The V-cluster is accompanied by a dipole, with a trough centered over Nova Scotia, and a ridge centered over Iceland. The trough coincides with anomalously high ETC activity.

[Figure 13 near here, please.]

The D-cluster and H-cluster are accompanied by opposite phases of the NAO (e.g., Hurrell et al. 2003), with north-south dipoles in geopotential height over the North Atlantic. The Dcluster corresponds to the positive phase of the NAO, with a trough over Greenland and a ridge to the south. In this phase, the NAO anomaly amplifies the climatological pressure gradients, leading to an intensified storm track over the North Atlantic and steering cyclones to the northeast. In 
the NAO's negative phase, the climatological gradients are weakened, so that cyclones tend to be weaker and track more zonally. Our results are consistent with the NAO index regressed onto root-mean-square transient geopotential height in the 2-8-day band (Hurrell et al. 2003, Fig. 15), which closely resembles our MSLP sub-weekly variance composites in Figs. 12g and h.

The most-populated cluster in both the reanalysis and the GCM data is the V-cluster; it is associated with a large-scale wave pattern that is less familiar from studies of low-frequency variability than the NAO. This pattern is indeed more transitory than those associated with the $\mathrm{D}$ and $\mathrm{H}$ clusters, with a larger number of run-lengths shorter than 5 days (not shown). It shares certain features with the Reverse W3 (RW3) wave train of Mo and Ghil (1988), the eastern Atlantic ridge (AR) of Vautard (1990), and ATL regime A2 of Kimoto and Ghil (1993). The differences consist mainly in a zonal shift of the main features and might be due to differences in the domain of analysis and data set, even more so than to the difference in the compositing. Vautard (1990) notes, in fact, that the storm track is both shortened and displaced northward for his AR regime.

\section{Summary and concluding remarks}

Curve-based mixture models were used to perform probabilistic clustering of wintertime North Atlantic extratropical cyclone (ETC) trajectories in latitude-longitude space. In contrast to previous 
clustering methods, trajectories have varying durations and the clustering is performed directly in "trajectory-space" rather than in a fixed-dimensional vector space. Quadratic polynomials were found to provide the best fits among the regression models we considered (Fig. 4).

An identification and tracking procedure using mean sea level pressure (MSLP) fields was developed and applied both to an NCAR CCM3 simulation and the NCEP-NCAR reanalysis, over the North Atlantic. The resulting cyclone trajectories (e.g., Fig. 1) were used as input to the clustering algorithm. The objective performance measures of log-likelihood and the sum of squared errors (Fig. 6) suggested that $K=3$ is a reasonable choice for the number of clusters, in both the GCM data and the reanalysis, resulting both cases in groups of tracks oriented predominantly south-to-north ("V"), southwest-to-northeast ("D”), and west-to-east ("H") respectively (Figs. 7 and 9). These three categories of tracks were found to share several attributes in both the GCM and reanalysis data (Figs. 8 and 9; Tables 1 and 2). The V-cluster consists of relatively short, slow-moving cyclones with S-N tracks, and intermediate curvature and noisiness. The D-cluster cyclones have the largest average velocity, intensity, and duration; their tracks are the straightest and smoothest of all cyclones. The H-cluster cyclones are relatively slow-moving and are the least intense on average, but have relatively large acceleration values, and the largest values of curvature and noisiness in both data sets. 
The main distinction between the GCM and reanalysis cyclones was found to be in their geographic distribution, with the reanalysis cyclones being much more geographically localized: the V-cluster cyclones over the eastern seaboard of North America, the D-cyclones in a narrower crossAtlantic swath, and the H-cluster more confined to the Atlantic and northwest Europe (Figs. 7 and 9). Composite maps of sub-weekly "storm track" MSLP variance anomalies were found to be quite consistent with the track distributions, again with the maps being much more spatially coherent in the reanalysis case (Fig. 12).

The clustering was performed solely by trajectory shape. Additional experiments (not shown) indicate that including initial position in the characterization of each ETC yields clusters with clearly defined geographical centers of gravity, but that the associated cluster composites of geopotential height are less amenable to physical interpretation.

Using $K$-mean analysis of 3-day-long tracks derived from 5 winters of higher-resolution ECMWF analysis, Blender et al. (1997) also obtained a total of 3 clusters. Of these, a cluster of nearstationary cyclones, concentrated over the Mediterranean and near Greenland, largely absent from our analysis; these cyclones are probably missed in our lower-resolution data set. Of these authors' two other clusters, the north-eastward one resembles our D-cluster, and their zonal cluster is quite similar to our H-cluster. Our heavily populated V-cluster, with most trajectories close to the coast- 
line of North America in the reanalysis data, differs from the results of Blender and colleagues.

The $\mathrm{D}$ and $\mathrm{H}$ clusters in the reanalysis were found to be closely related to the opposite phases of the well-known NAO teleconnection pattern (Fig. 13c,d). The positive phase of the NAO is associated with diagonally oriented tracks with cyclones that are typically faster, more intense, of longer duration, and with the straightest and smoothest tracks. This contrasts with the horizontally oriented tracks that characterize the NAO's negative phase, which are typically weaker, moving more slowly, and fairly erratic. This association with the phases of the NAO arises out of our clustering that is based purely on trajectory shape. We conclude that this statistical association does have a physical explanation in terms of the dynamical features of the opposite NAO phases.

The most highly populated V-cluster was found to be associated in the reanalysis with a trough over the western and a ridge over the eastern North Atlantic (Fig. 13b). This large-scale feature has been identified by various names in different studies: RW3 in Mo and Ghil (1988), AR in Vautard (1990), and A2 in Kimoto and Ghil (1993). The ridge blocks the eastward propagation of the ETCs, while the trough favors their northward evolution. To summarize, the ease of comparison between GCM clusters and those in the reanalysis, as well as the physical interpretation of the latter, support the choice of three clusters as a good coarse-grained description of ETC behavior over the North Atlantic. We note, furthermore, that these three clusters also agree with three of 
the four regimes obtained by Yiou and Nogaj (2004) in classifying extremes of precipitation and temperature over a similar area.

Having demonstrated that, in reanalysis data, the cyclone-track clusters are associated with well-defined anomalies in sub-weekly storm track variance and well-known low-frequency teleconnection patterns, we argue that further analysis of ETC track behavior from the Lagrangian perspective used in this paper could enable a more fundamental interpretation of these features. Unlike in the case of the reanalysis data, meteorological composites constructed from the 15winter GCM simulation did not provide conclusive evidence for associations between ETC track behavior and large-scale circulation patterns in the simulation. The methodology clearly highlights the limitations of the GCM, while the GCM is shown nonetheless to capture cyclone tracks of quite realistic orientation, as well as several associated features of cyclone intensity, speed and lifetimes. Lagrangian diagnostics could thus provide important tools in assessing GCM performance for studies of climate variability and change. The method has, therefore, also been applied to clustering of tropical cyclone tracks over the western tropical North Pacific (Camargo et al. 2006a,b). The software developed and used in this study is freely available to other investigators from http://www.datalab.uci.edu/resources/CCT/. We hope that the methodology will prove useful in further studies of ETC behavior in models and observations. 
Acknowledgments: We wish to thank Kevin Hodges for helpful discussions, and Jim Boyle and Peter Glecker for help in obtaining the NCAR CCM3 data. We are grateful to Kevin Hodges and two anonymous referees for their constructive reviews which substantially improved the paper. The NCEP-NCAR Reanalysis data were provided by the NOAA CIRES Climate Diagnostics Center, Boulder, Colorado, from their Web site available online at http://www.cdc.noaa.gov. This work was supported in part by a Department of Energy grant DE-FG02-02ER63413 (MG and AWR), by NOAA through a block grant to the International Research Institute for Climate and Society (SJC and AWR), and by the National Science Foundation under grants No. SCI-0225642, IIS-0431085, and ATM-0530926 (SJG and PS).

\section{Appendix A}

\section{Expectation Maximization Algorithm}

The EM algorithm is an iterative maximum likelihood (ML) procedure that provides a general and efficient framework for parameter estimation. At a base level, EM is an approximate root-finding procedure used to seek the root of the likelihood equation by iteratively searching for a set of parameters that maximize the probability of the observed data. EM is primarily used for finding 
ML parameter estimates in missing- or hidden-data problems. Parameter estimation in hidden-data problems is difficult because the likelihood equation takes on a complex form, often involving an integral or a sum over the hidden data itself.

For example, Eqn. (5) in Sect. 3.c gives the likelihood of $\phi$ given both $Z$ and $\boldsymbol{T}$ (repeated here):

$$
L(\phi \mid \boldsymbol{Z}, \boldsymbol{T})=p(\boldsymbol{Z} \mid \boldsymbol{T}, \phi)=\prod_{i}^{n} \sum_{k}^{K} \alpha_{k} f_{k}\left(\mathbf{z}_{i} \mid \mathbf{T}_{i} \boldsymbol{\beta}_{k}, \Sigma_{k}\right)
$$

Notice that the hidden data in this case are the unknown cluster memberships which must be summed-out of the likelihood to arrive at $L(\phi \mid \boldsymbol{Z}, \boldsymbol{T})$. It is understood in hidden-data problems that this operation cannot be easily carried out. The EM algorithm is an iterative two-step procedure used to circumvent this integration (or sum) by (1) indirectly estimating values for the unobserved data, and (2) finding the ML parameter estimates that correspond to the now completely observed data. The new ML estimates from step (2) are then used to re-estimate the hidden data in step (1), and these iterations are continued until some stopping criterion is reached (typically this involves stopping when the change in log-likelihood falls below a particular threshold, and thus the iterations have stabilized).

In the first step, the E-step, we estimate the hidden cluster memberships by forming the ratio 
of the likelihood of trajectory $i$ under cluster $k$, to the sum-total likelihood of trajectory $i$ under all

clusters:

$$
w_{i k}=\frac{\alpha_{k} f_{k}\left(\mathbf{z}_{i} \mid \mathbf{T}_{i} \boldsymbol{\beta}_{k}, \Sigma_{k}\right)}{\sum_{j}^{K} \alpha_{j} f_{j}\left(\mathbf{z}_{i} \mid \mathbf{T}_{i} \boldsymbol{\beta}_{j}, \Sigma_{j}\right)}
$$

These $w_{i k}$ give the probabilities that the $i$-th trajectory was generated from cluster $k$. They represent a posterior expectation for the value of the actual binary cluster memberships (i.e., the $i$-th trajectory was either generated by the $k$-th cluster or it was not).

In the second step, the M-step, the expected cluster memberships from the E-step are used to form the weighted log-likelihood function:

$$
\mathcal{L}(\phi \mid \boldsymbol{Z}, \boldsymbol{T})=\sum_{i}^{n} \sum_{k}^{K} w_{i k} \log \alpha_{k} f_{k}\left(\mathbf{z}_{i} \mid \mathbf{T}_{i} \boldsymbol{\beta}_{k}, \Sigma_{k}\right)
$$

The membership probabilities weight the contribution that the $k$-th density component adds to the overall likelihood. In the case where the $w_{i k}$ are binary, and thus cluster membership is perfectly known, this reduces to the usual fully-observed log-likelihood. This weighted log-likelihood is then maximized with respect to the parameter set $\phi$.

For the sake of completeness, we give each of the re-estimation equations below. Let $\mathbf{w}_{i k}=$ $w_{i k} \mathbf{I}_{n_{i}}$, where $\mathbf{I}_{n_{i}}$ is an $n_{i}$-vector of ones, and let $\mathbf{W}_{k}=\operatorname{diag}\left(\mathbf{w}_{1 k}^{\prime}, \ldots, \mathbf{w}_{n k}^{\prime}\right)$ be an $N \times N$ diagonal 
matrix. Then, in the M-step we use $\mathbf{W}_{k}$ to calculate the mixture parameters

$$
\begin{aligned}
& \hat{\boldsymbol{\beta}}_{k}=\left(\mathbf{T}^{\prime} \mathbf{W}_{k} \mathbf{T}\right)^{-1} \mathbf{T}^{\prime} \mathbf{W}_{k} \mathbf{Z}, \\
& \hat{\Sigma_{k}}=\frac{\left(\mathbf{Z}-\mathbf{T} \hat{\beta}_{k}\right)^{\prime} \mathbf{W}_{k}\left(\mathbf{Z}-\mathbf{T} \hat{\beta}_{k}\right)}{\sum_{i}^{n} w_{i k}},
\end{aligned}
$$

and the mixture weights

$$
\hat{\alpha_{k}}=\frac{1}{n} \sum_{i}^{n} w_{i k}
$$

for $k=1, \ldots, K$. These update equations are equivalent to the well-known weighted least-squares solution in regression (Draper and Smith 1981). The diagonal elements of $\mathbf{W}_{k}$ represent the weights to be applied to $\mathbf{Z}$ and $\mathbf{T}$ during the weighted regression.

Because most of the difficult work is carried out in estimating the cluster memberships, the maximization carried out in the M-step is straightforward. This is a common attribute of the EM algorithm. Dempster et al. (1977b) showed that under fairly general conditions, the likelihood will never decrease during the E- and M-step iterations. Due to the presence of local maxima on the likelihood surface, the solution is not guaranteed to correspond to a global maximum. However, 
we can increase the chances of finding the global maximum by running the EM algorithm multiple times from different starting points in parameter space and selecting the parameters that result in the highest overall likelihood. 


\section{References}

Anderson D, Hodges KI, Hoskins BJ (2003) Sensitivity of Feature-Based Analysis Methods of

Storm Tracks to the Form of Background Field. Mon Wea Rev 131(3):565-573

Bernardo JM, Smith AFM (1994) Bayesian Theory, Wiley, New York

Blackmon ML, Wallace JM, Lau NC, Mullen SL (1977) An Observational Study of the Northern Hemisphere Wintertime Circulation. J Atmos Sci 34:1040-1053

Blender R, Fraedrich K, Lunkeit F (1997) Identification of cyclone-track regimes in the North Atlantic. Quart J Royal Meteor Soc 123:727-741

Camargo SJ, Robertson AW, Gaffney SJ, Smyth P (2006a) Cluster Analysis of Typhoon Tracks. Part I: General Properties. J Climate in press

Camargo SJ, Robertson AW, Gaffney SJ, Smyth P (2006b) Cluster Analysis of Typhoon Tracks. Part II: Large-Scale Circulation and ENSO. J Climate in press

Dempster AP, Laird NM, Rubin DB (1977a) Maximum likelihood from incomplete data via the EM algorithm. J Royal Stat Soc B 39:1-38 
Dempster AP, Laird NM, Rubin DB (1977b) Maximum likelihood from incomplete data via the EM algorithm. J Royal Stat Soc B 39:1-38

DeSarbo WS, Cron WL (1988) A maximum likelihood methodology for clusterwise linear regression. J Classification 5(1):249-282

Draper NR, Smith H (1981) Applied Regression Analysis, John Wiley and Sons, New York, NY, 2nd ed.

Elsner JB (2003) Tracking hurricanes. Bull Amer Meteor Soc 84(3):353-356

Elsner JB, b Liu K, Kocher B (2000) Spatial variations in major US hurricane activity: Statistics and a physical mechanism. J Climate 13:2293-2305

Fraley C, Raftery AE (1998) How many clusters? Which clustering methods? Answers via modelbased cluster analysis. The Computer Journal 41(8):578-588

Fraley C, Raftery AE (2002) Model-based clustering, discriminant analysis, and density estimation. J Amer Stat Assoc 97(458):611-631

Fyfe JC (2003) Extratropical Southern Hemisphere Cyclones: Harbingers of Climate Change. J Climate 16:2802-2805 
Gaffney S, Smyth P (1999) Trajectory Clustering with Mixtures of Regression Models, in S Chaudhuri, D Madigan, (eds.) Proc. Fifth ACM SIGKDD Internat. Conf. on Knowledge Discovery and Data Mining, pp. 63-72, ACM Press, N.Y.

Gaffney SJ (2004) Probabilistic Curve-Aligned Clustering and Prediction with Regression Mixture Models, Ph.D. Dissertation, Department of Computer Science, University of California, Irvine

Gaffney SJ, Smyth P (2003) Curve Clustering with Random Effects Regression Mixtures, in CM Bishop, BJ Frey, (eds.) Proc. 9th Internat. Workshop on Artificial Intelligence and Statistics, Key West, FL

Gneiting T, Raftery AE (2004) Strictly proper scoring rules, prediction, and estimation, Tech. Rep. 463, Department of Statistics, University of Washington

Hack JJ, Kiehl JT, J. W. H (1998) The Hydrologic and Thermodynamic Characteristics of the NCAR CCM3. J Climate 11:1179-1206

Hannachi A, O’Neill A (2001) Atmospheric multiple equilibria and non-Gaussian behaviour in model simulations. Quart J Royal Meteor Soc 127(573):939-958

Hartigan JA, Wong MA (1978) Algorithm AS 136: A K-means clustering algorithm. Appl Stat 28:100-108 
Hodges KI (1994) A general method for tracking analysis and its applications to meteorological data. Mon Wea Rev 122(11):2573-2586

Hodges KI (1995) Feature tracking on the unit sphere. Mon Wea Rev 123(12):3458-3465

Hodges KI (1998) Feature-point detection using distance transforms: Application to tracking tropical convective complexes. Mon Wea Rev 126(3):785-795

Hoskins BJ, Hodges KI (2002) New Perspectives on the Northern Hemisphere Winter Storm Tracks. J Atmos Sci 59(6):1041-1061

Hurrell JW, Kushnir Y, Ottersen G, Visbeck M (2003) An overview of the North Atlantic Oscillation. Geophys Monogr 134:2217-2231

Kalnay E, Kanamitsu M, Kistler R, Collins W, Deaven D, Gandin L, Iredell M, Saha S, White G, Woollen J, Zhu Y, Chelliah M, Ebisuzaki W, Higgins W, Janowiak J, Mo K, Ropelewski C, Wang J, Leetmaa A, Reynolds R, Jenne R, Joseph D (1996) The NCEP/NCAR 40-year reanalysis project. Bull Amer Meteor Soc 77:437-441

Kimoto M, Ghil M (1993) Multiple Flow Regimes in the Northern Hemisphere Winter. Part II: Sectorial Regimes and Preferred Transitions. J Atmos Sci 16:2645-2673 
König W, Sausen R, Sielman F (1993) Objective identification of cyclones in GCM simulations. J Climate 6(12):2217-2231

Lau NC (1988) Variability of the Observed Midlatitude Storm Tracks in Relation to LowFrequency Changes in the Circulation Pattern. J Atmos Sci 45:2718-2743

Le Treut H, Kalnay E (1990) Comparison of observed and simulated cyclone frequency distribution as determined by an objective method. Atmosfera 3:57-71

Lenk PJ, DeSarbo WS (2000) Bayesian inference for finite mixtures of generalized linear models with random effects. Psychometrika 65(1):93-119

Lwin T, Martin PJ (1989) Probits of mixtures. Biometrics 45:721-732

Mailier PJ, Stephenson DB, Ferro CAT, Hodges KJ (2006) Serial Clustering of Extratropical Cyclones. Monthly Weather Review 134:2224-2240

McLachlan G, Krishnan T (1997) The EM Algorithm and Extensions, John Wiley and Sons, New York, NY

McLachlan GJ, Basford KE (1988) Mixture Models: Inference and Applications to Clustering, Marcel Dekker, New York 
McLachlan GJ, Peel D (2000) Finite Mixture Models, John Wiley \& Sons, New York

Mesrobian E, Muntz R, Shek E, Mechoso CR, Farrara J, Spahr J, Stolorz P (1995) Real Time Data Mining, Management, and Visualization of GCM Output. Supercomputing 94 IEEEComputerSociety pp. $81-87$

Mo K, Ghil M (1988) Cluster analysis of multiple planetary flow regimes. J Geophys Res 93D:10927-10952

MunichRe (2002) Winter storms in Europe (II). Analysis of 1999 losses and loss potentials, Tech. Rep. Rep. 302-03109, 72pp, [Available from Münchner Rückversicherungs-Gesellschaft, Königinstr. 107, 80802 München, Germany.]

Murray RJ, Simmonds I (1991) A numerical scheme for tracking cyclone centres from digital data Part I: Development and operation of the scheme. Aust Meteor Mag 39:155-166

Preisendorfer RW (1988) Principal Component Analysis in Meteorology and Oceanography, Elsevier, Amsterdam

Ramsay JO, Silverman BW (1997) Functional Data Analysis, Springer-Verlag, New York, NY

Ramsay JO, Silverman BW (2002) Applied Functional Data Analysis: Methods and Case Studies, Springer-Verlag, New York, NY 
Robertson AW, Metz W (1990) Transient-Eddy Feedbacks Derived from Linear Theory and Observations. J Atmos Sci 47:2743-2764

Saunders MA (1999) Earth's future climate. Phil Trans Royal Soc Lond A 357:3459-3480

Simmons AJ, Hoskins BJ (1978) The Life Cycles of Some Nonlinear Baroclinic Waves. J Atmos Sci 35(3):414-432

Smyth P (2000) Model selection for probabilistic clustering using cross-validated likelihood. Statistics and Computing 10(1):63-72

Smyth P, Ide K, Ghil M (1999) Multiple regimes in northern hemisphere height fields via mixture model clustering. J Atmos Sci 56(21):3704-3723

Terry J, Atlas R (1996) Objective Cyclone Tracking and its Applications to ERS-1 Scatterometer Forecast Impact Studies, in 15th Conf. Weather Analysis \& Forecasting, American Meteorological Society, Norfolk, VA.

Trenberth KE (1986) An Assessment of the Impact of Transient Eddies on the Zonal Flow during a Blocking Episode Using Localized Eliassen-Palm Flux Diagnostics. J Atmos Sci 43:2070-2087

Vautard R (1990) Multiple Weather Regimes over the North Atlantic: Analysis of Precursors and Successors. Mon Wea Rev 45:2845-2867 
von Storch H, Zwiers FW (1999) Statistical Analysis in Climate Research, Cambridge University Press, Cambridge, MA

Vrac M, Chedin A, Diday E (2005) Clustering a Global Field of Atmospheric Profiles by Mixture Decomposition of Copulas. Journal of Atmospheric and Oceanic Technology 22(10):1445-1459

Wang K, Gasser T (1997) Alignment of curves by dynamic time warping. Annal Stat 25:12511276

Yiou P, Nogaj M (2004) Extreme climatic events and weather regimes over the North Atlantic: When and where? Geophys Res Lett 31:L07202, doi:10.1029/2003GL019119 


\section{List of Figures}

1 Random sample of 200 CCM3 cyclone trajectories tracked over the North Atlantic domain of interest. The circles indicate initial starting position. . . . . . . . . . 54

2 Summary histograms for GCM cyclone data set: (a) cyclone duration, (b) average velocity, and (c) maximum intensity (MSLP) . . . . . . . . . . . . . . . 55

3 Performance of the EM algorithm as applied to synthetic trajectories, generated by a polynomial-regression mixture model: (a) set of synthetic trajectories presented to the algorithm (the x-es, circles, and squares denote the 3 generating models, and the different line-types show the 4 different sample curves for each); (b) initial random starting curves (solid) for the three clusters, with all data points shown as circles; (c) cluster locations (solid) after EM convergence (iteration 4), as well as the locations of the true data-generating trajectories (dotted) . . . . . . . 56

4 Quadratic polynomial regression models (dotted) fitted to a random sample of three GCM cyclone trajectories (solid) in the latitude-time plane. . . . . . . . . . . . 57

$5 \quad$ GCM cyclone cluster models when $K=2,3,4,5$, and $6 \ldots \ldots \ldots 8$ 
6 Objective test scores of model fit as a function of $K$ for GCM cyclone cluster models. (a) Cross-validated log-likelihood; and (b) cross-validated sum of squared errors (SSE). See text for details. . . . . . . . . . . . . . . . . . . . . . . 59

7 Clusters derived from GCM data: (V) south-to-north, (D) southwest-to-northeast, and $(\mathrm{H})$ west-to-east oriented tracks. For each cluster only 100 random tracks are shown for clarity. . . . . . . . . . . . . . . . . . 60

8 Histograms stratified by GCM cluster: (a) average velocity, (b) cyclone duration, and (c) maximum intensity (MSLP). The histograms are plotted as line graphs for

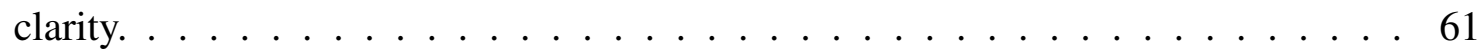

9 Clusters derived from reanalysis data: (V) south-to-north, (D) southwest-to-northeast, and $(\mathrm{H})$ west-to-east. For each cluster 100 random tracks are shown for clarity. . . 62

10 Histograms of reanalysis trajectories stratified by cluster: (a) average velocity, (b) cyclone duration, and (c) maximum intensity (MSLP). The histograms are plotted

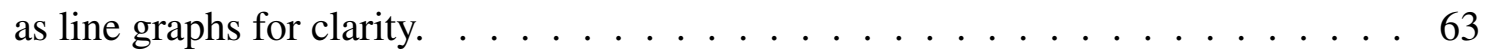

11 Mean climatologies of high-pass filtered MSLP variance for the (a) GCM and (b) reanalysis data sets. Contour interval: $5 \mathrm{hPa}^{2} \ldots \ldots \ldots \ldots$ 
12 Composites of high-pass filtered MSLP variance anomalies over the days assigned to each cluster, for the GCM (a-d) and reanalysis (e-h) data sets. In each case the respective climatological time average of variance has been subtracted. Positive contours are solid and negative ones are dashed; contour interval: $1 \mathrm{hPa}^{2} . \ldots . .65$

13 Composites of low-pass filtered 700-hPa geopotential height anomalies for the days assigned into each reanalysis cluster. In each case the 44-winter time average has been subtracted. The shaded regions are significant at the 99\% level according to a two-sided Student $t$-test with 120 degrees of freedom; this number is smaller than the number of days in each composite divided by 10. Contour interval: $5 \mathrm{~m} . \quad$. 66 


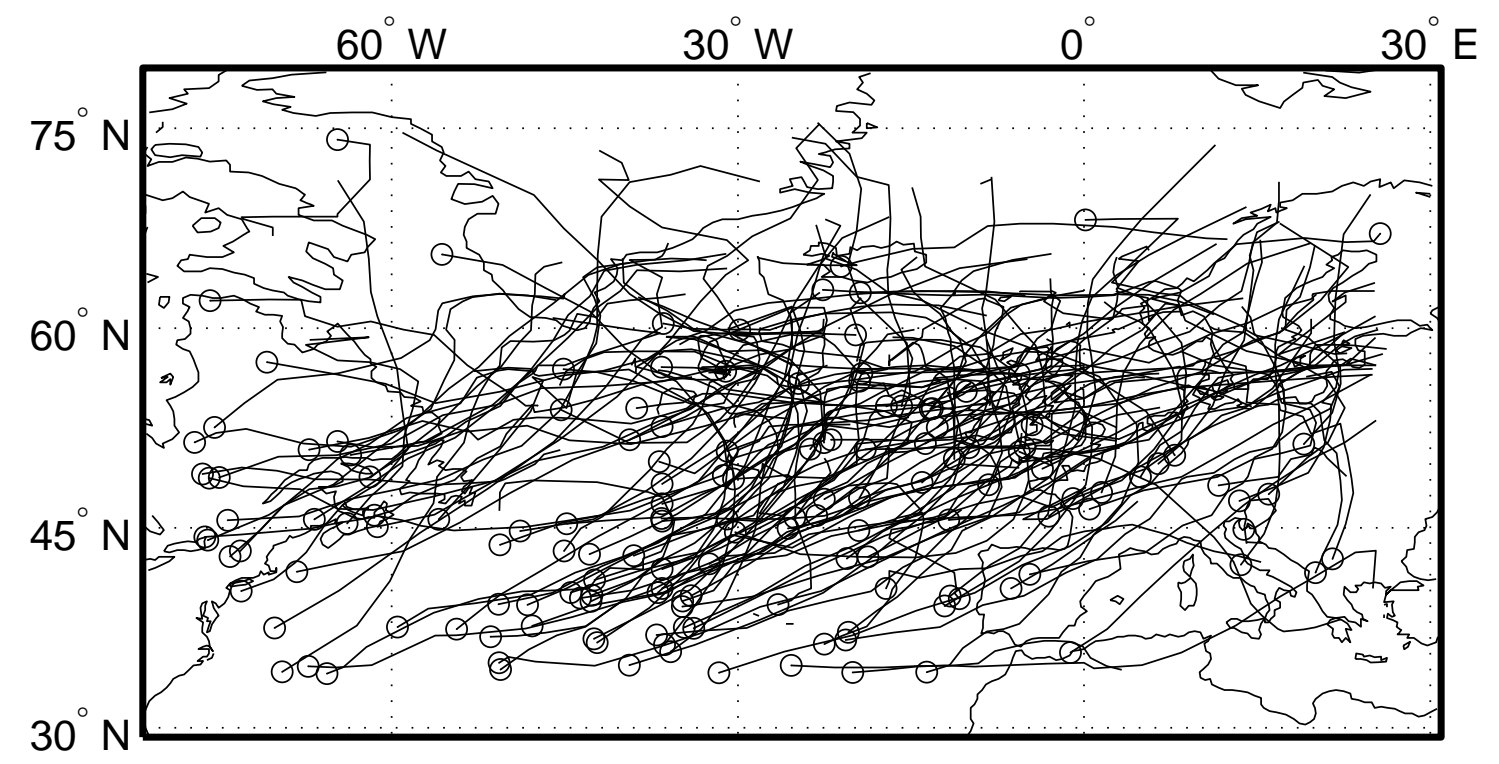

Figure 1. Random sample of 200 CCM3 cyclone trajectories tracked over the North Atlantic domain of interest. The circles indicate initial starting position. 

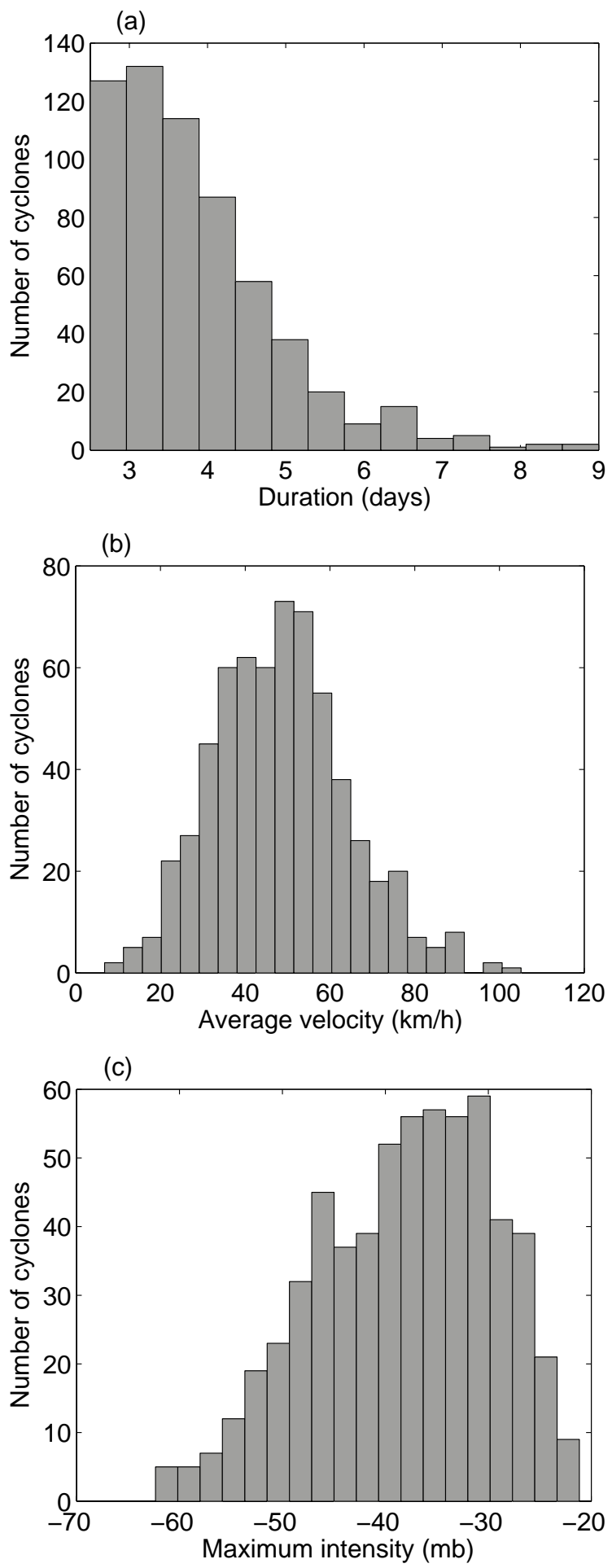

Figure 2. Summary histograms for GCM cyclone data set: (a) cyclone duration, (b) average velocity, and (c) maximum intensity (MSLP). 

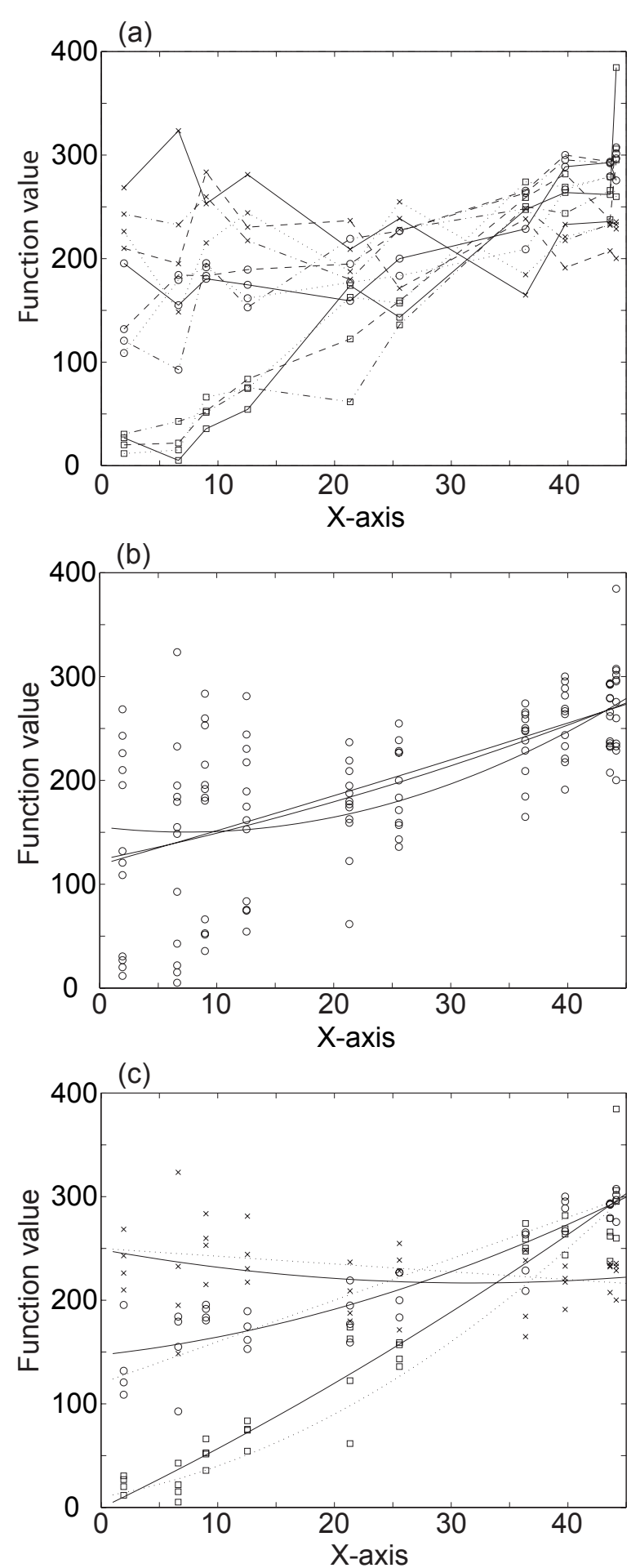

Figure 3. Performance of the EM algorithm as applied to synthetic trajectories, generated by a polynomial-regression mixture model: (a) set of synthetic trajectories presented to the algorithm (the $\mathrm{x}$-es, circles, and squares denote the 3 generating models, and the different line-types show the 4 different sample curves for each); (b) initial random starting curves (solid) for the three clusters, with all data points shown as circles; (c) cluster locations (solid) after EM convergence (iteration 4), as well as the locations of thetrue data-generating trajectories (dotted). 


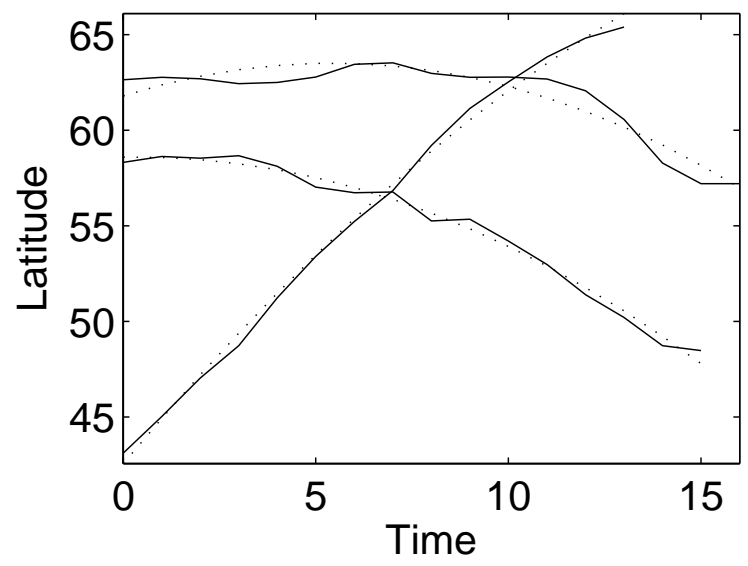

Figure 4. Quadratic polynomial regression models (dotted) fitted to a random sample of three GCM cyclone trajectories (solid) in the latitude-time (days) plane. 

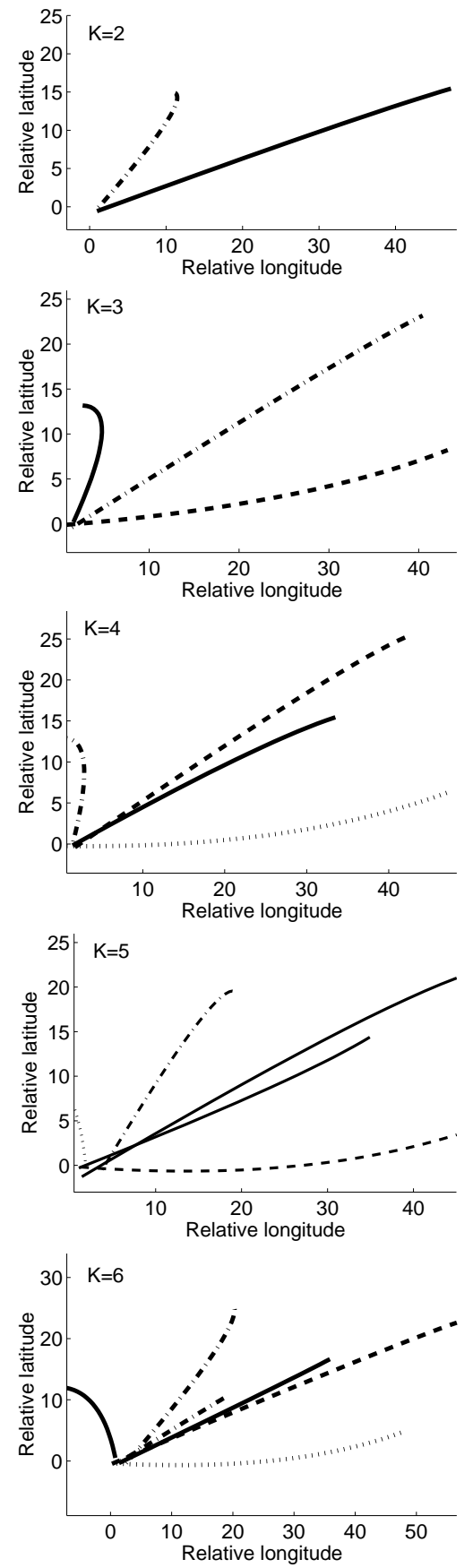

Figure 5. GCM cyclone cluster models when $K=2,3,4,5$, and 6 . 

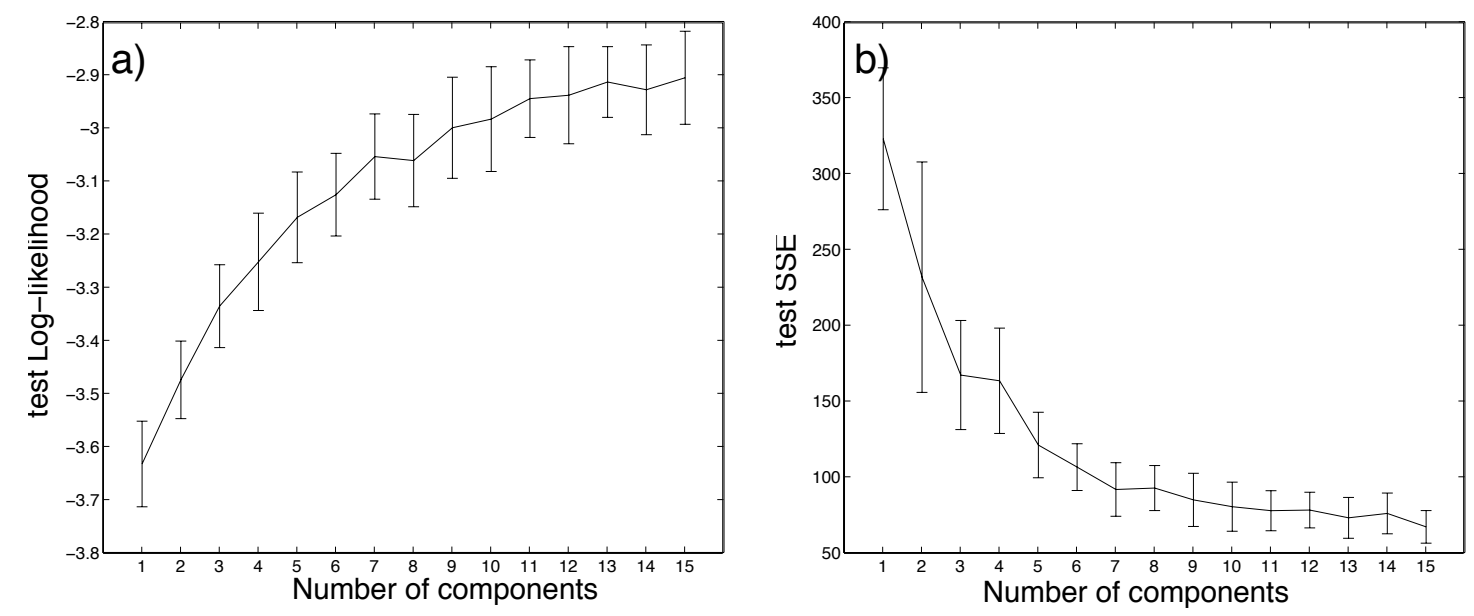

Figure 6. Objective test scores of model fit as a function of $K$ for GCM cyclone cluster models. (a) Cross-validated log-likelihood; and (b) cross-validated sum of squared errors (SSE). See text for details. 

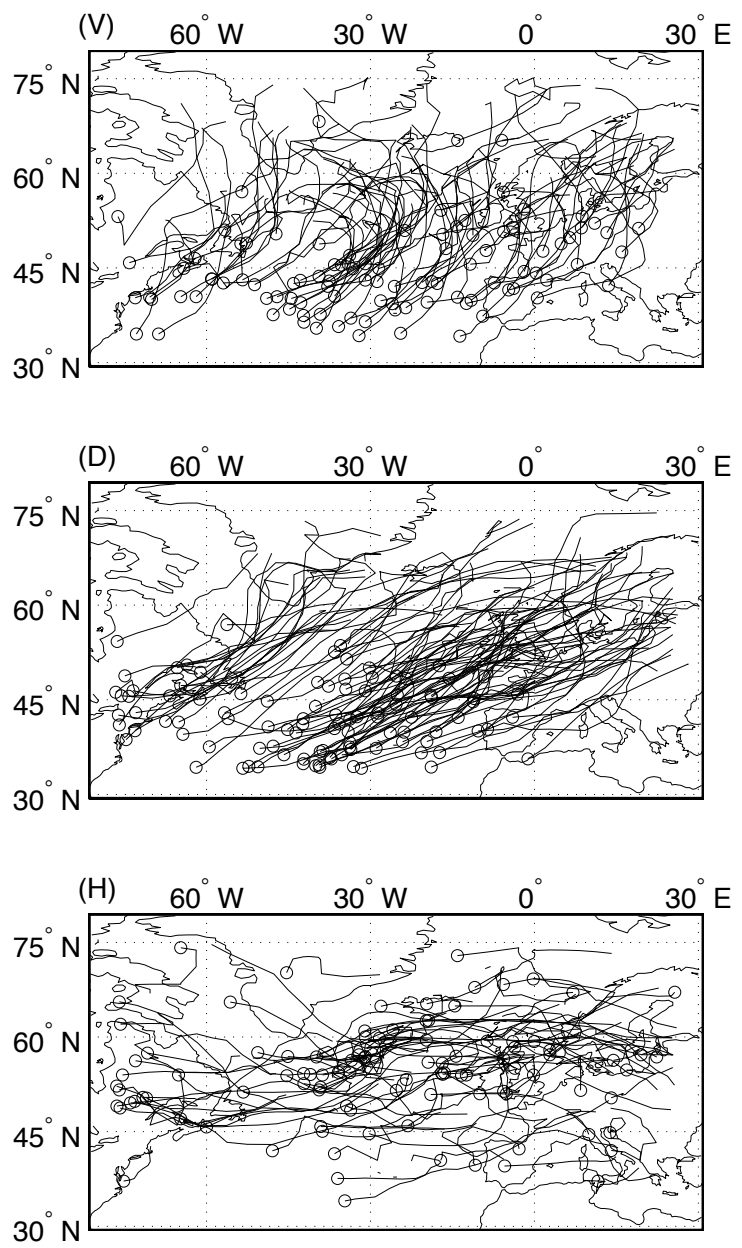

Figure 7. Clusters derived from GCM data: (V) south-to-north, (D) southwest-to-northeast, and (H) west-to-east oriented tracks. For each cluster only 100 random tracks are shown for clarity. 
(a)
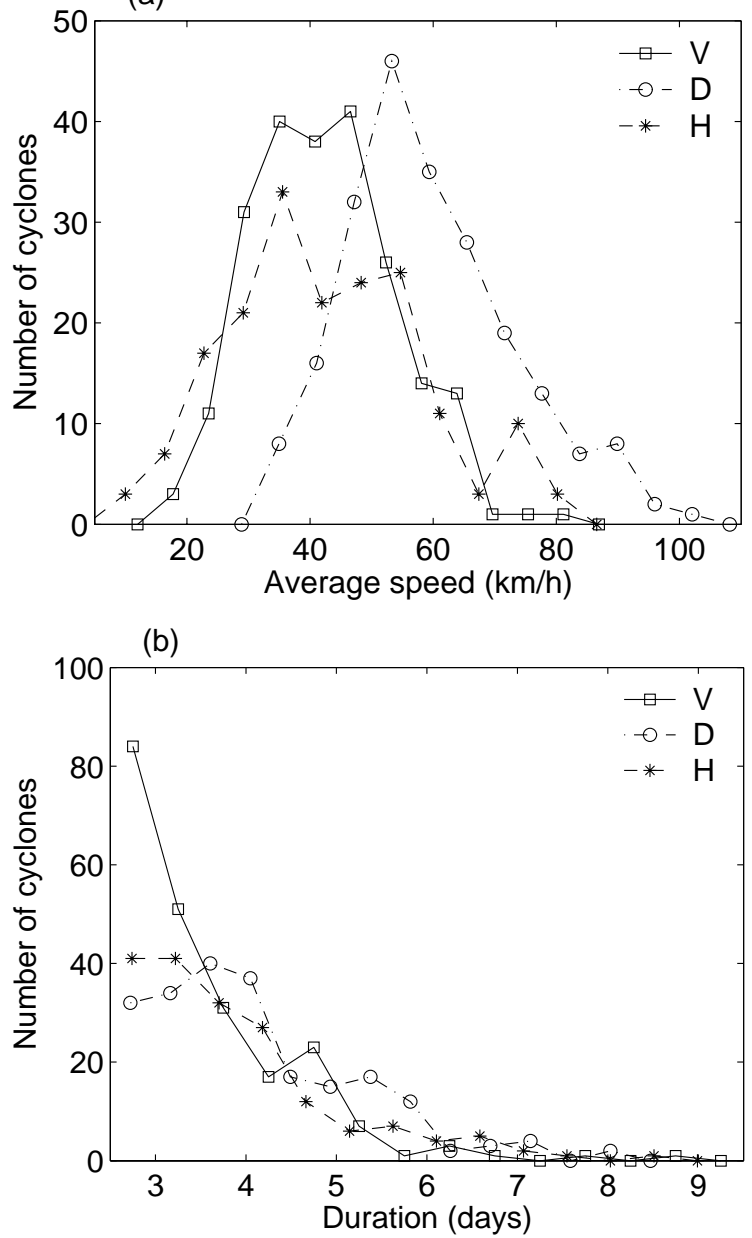

(c)

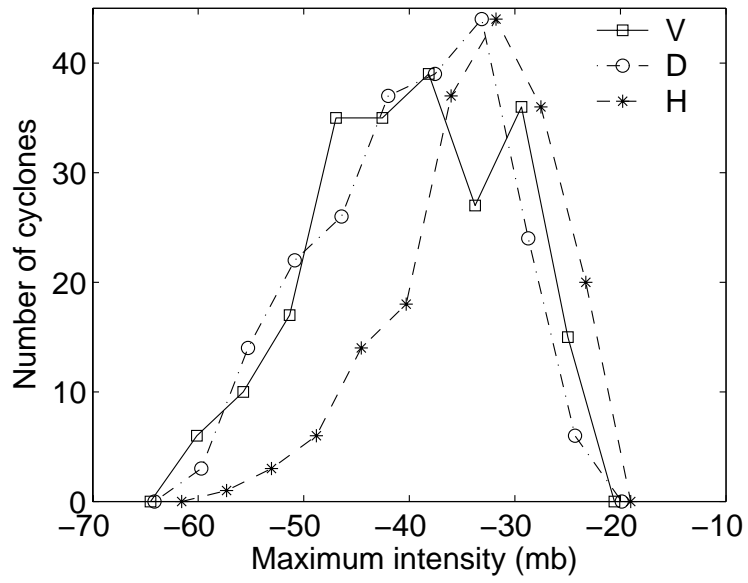

Figure 8. Histograms stratified by GCM cluster: (a) average velocity, (b) cyclone duration, and (c) maximum intensity (MSLP). The histograms are plotted as line graphs for clarity. 

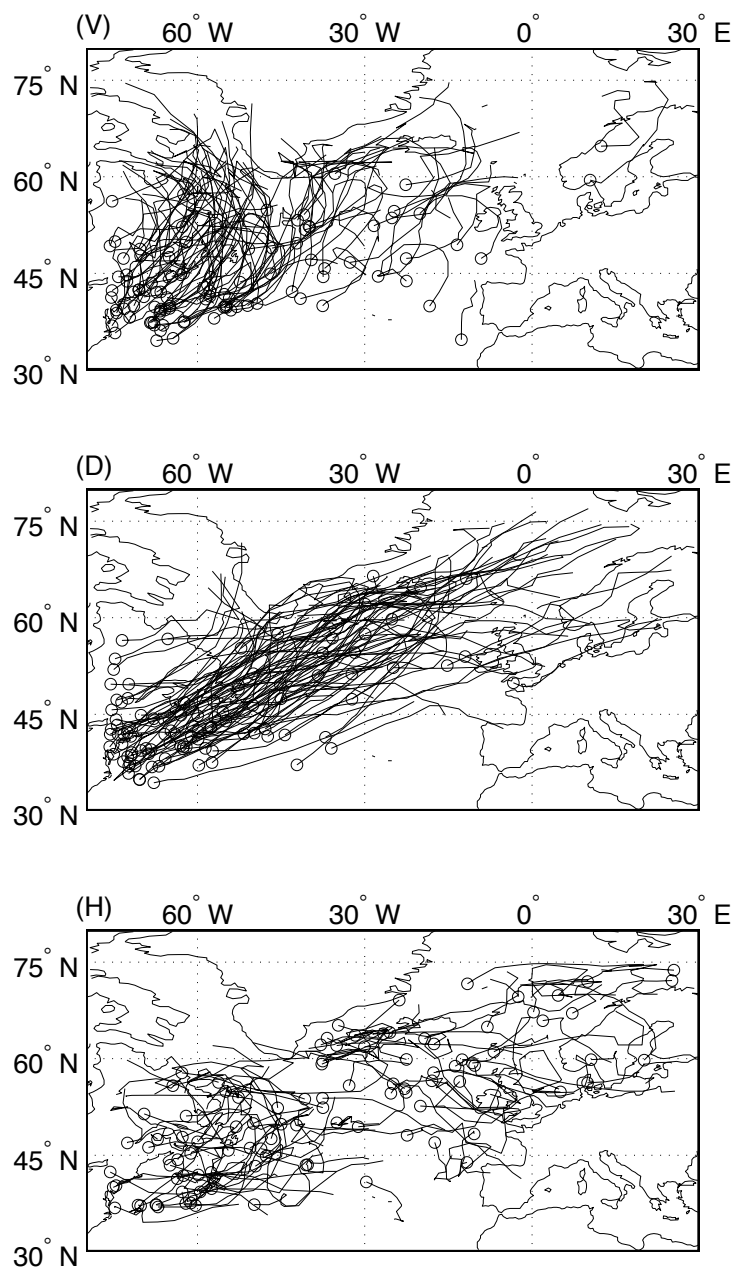

Figure 9. Clusters derived from reanalysis data: (V) south-to-north, (D) southwest-to-northeast, and $(\mathrm{H})$ west-to-east. For each cluster 100 random tracks are shown for clarity. 

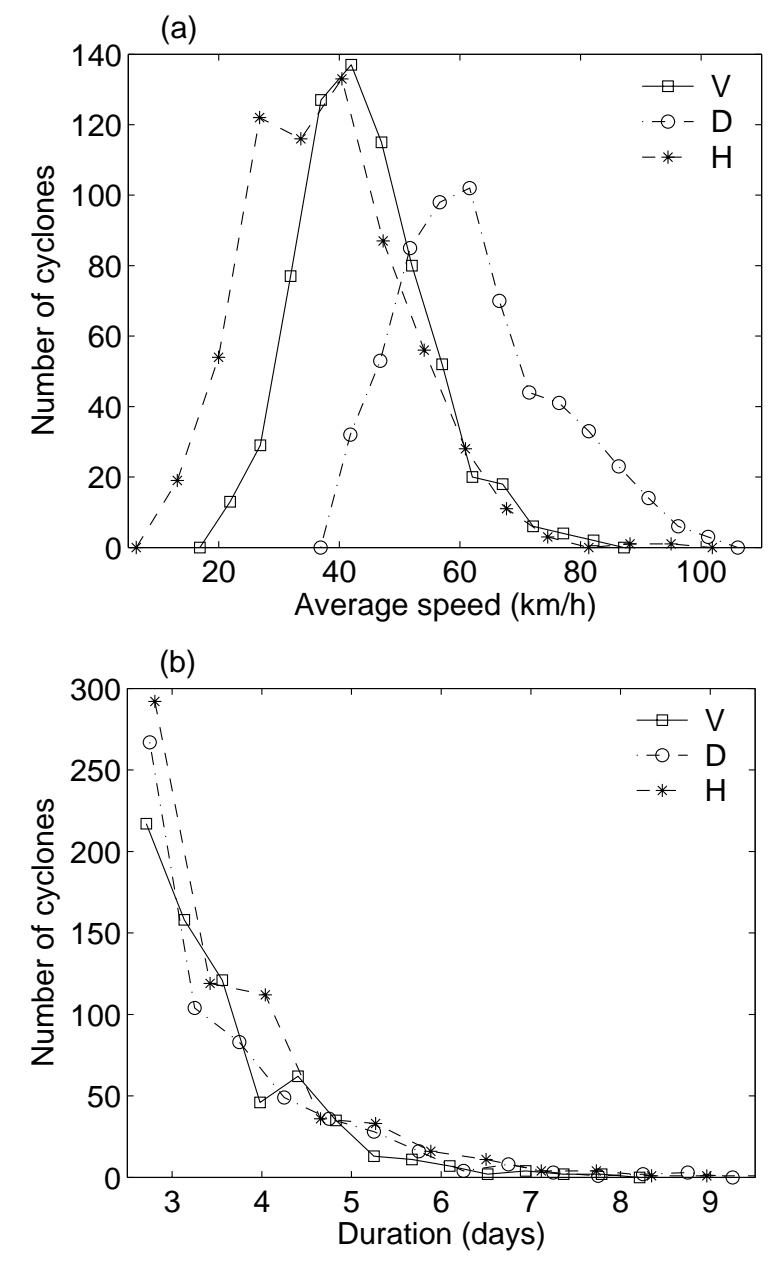

(c)

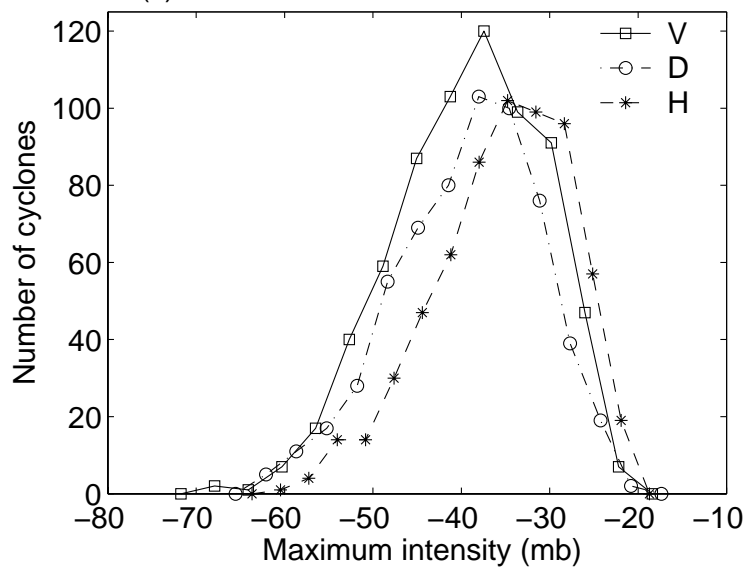

Figure 10. Histograms of reanalysis trajectories stratified by cluster: (a) average velocity, (b) cyclone duration, and (c) maximum intensity (MSLP). The histograms are plotted as line graphs for clarity. 
a) GCM High-pass Variance

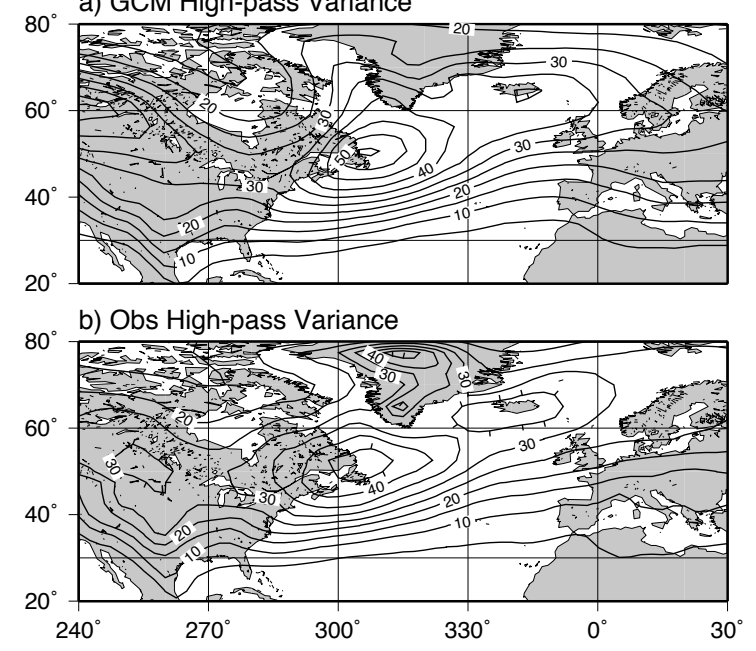

Figure 11. Mean climatologies of high-pass filtered MSLP variance for the (a) GCM and (b) reanalysis data sets. Contour interval: $5 \mathrm{hPa}^{2}$. 


\section{GCM}
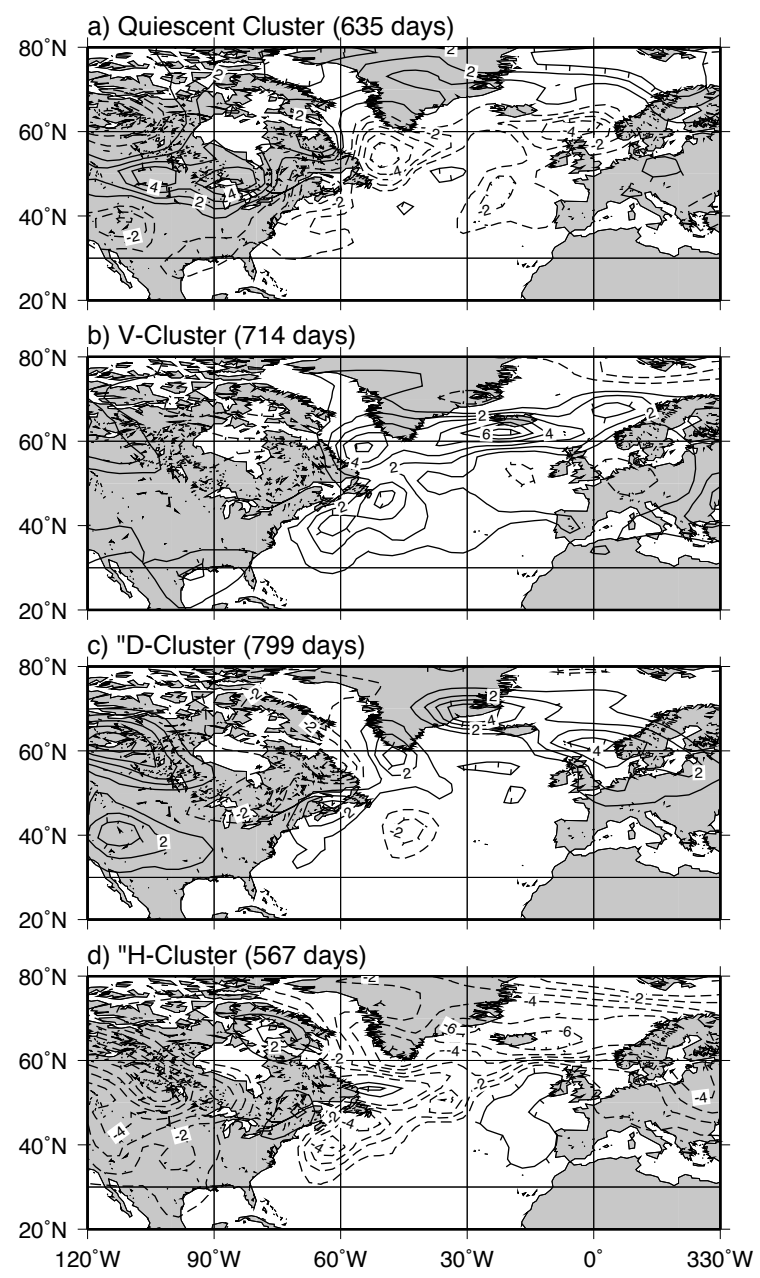

Reanalysis
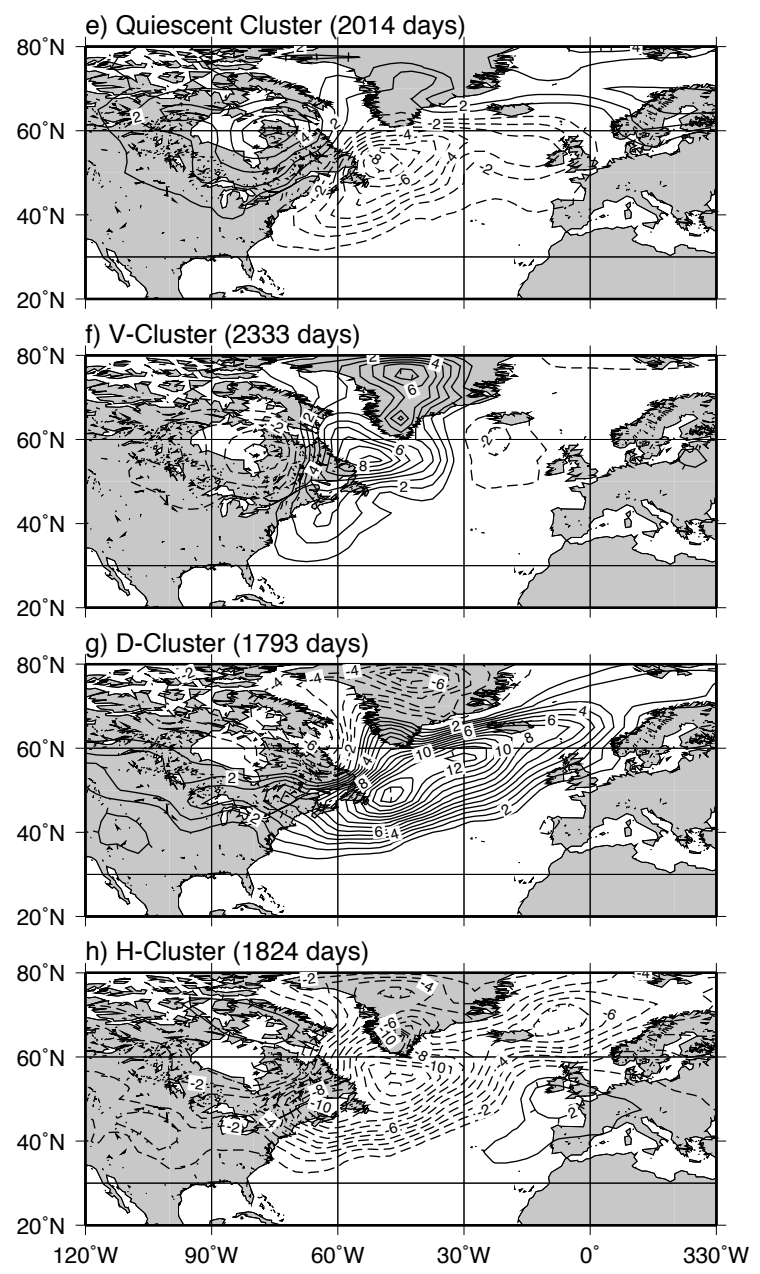

Figure 12. Composites of high-pass filtered MSLP variance anomalies over the days assigned to each cluster, for the GCM $(\mathrm{a}-\mathrm{d})$ and reanalysis $(\mathrm{e}-\mathrm{h})$ data sets. In each case the respective climatological time average of variance has been subtracted. Positive contours are solid and negative ones are dashed; contour interval: $1 \mathrm{hPa}^{2}$. 
a) Quiescent Cluster (2014 days)

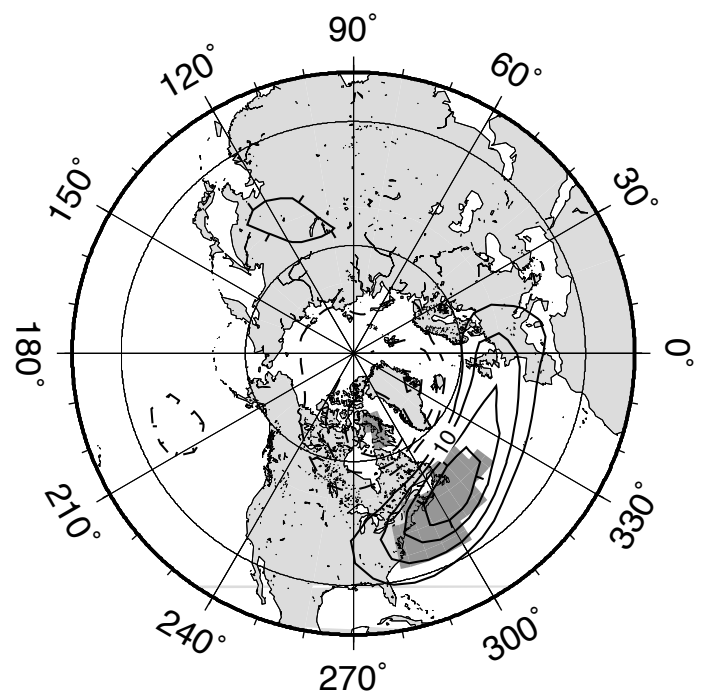

c) D-Cluster (1793 days)

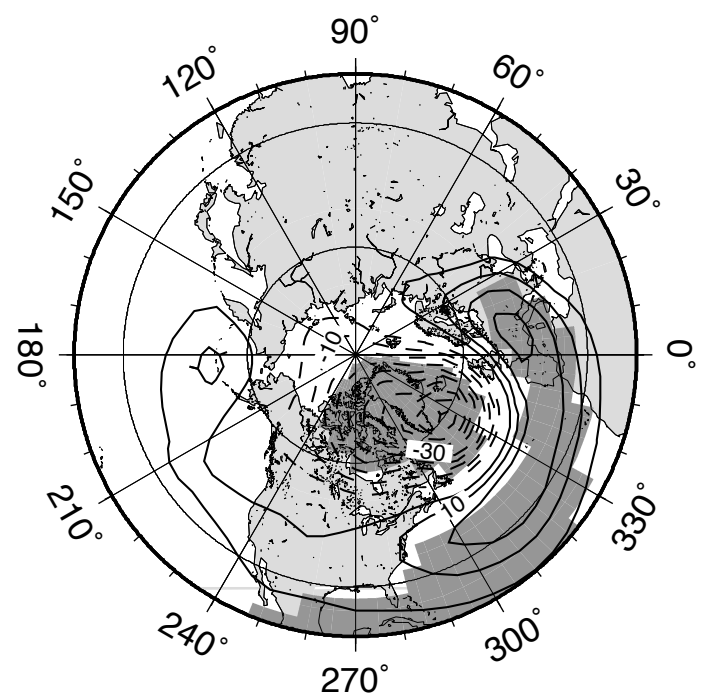

b) V-Cluster (2333 days)

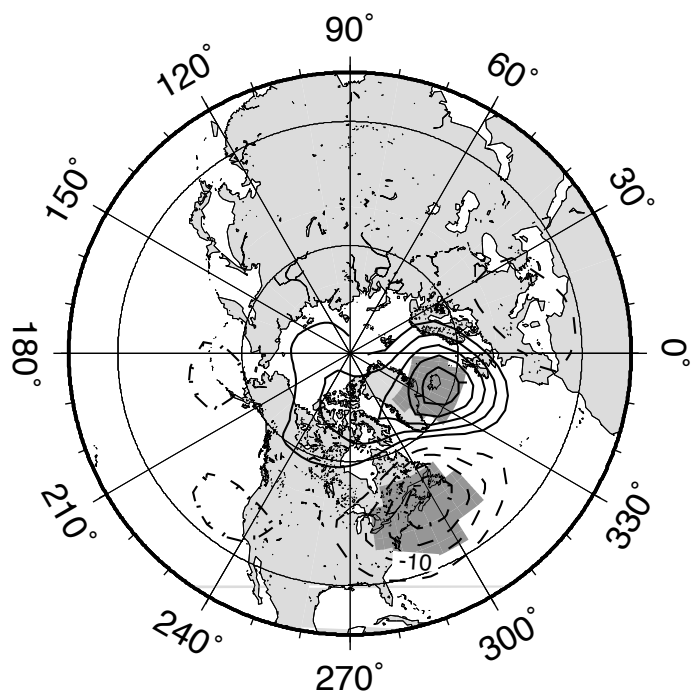

d) $\mathrm{H}$-Cluster (1824 days)

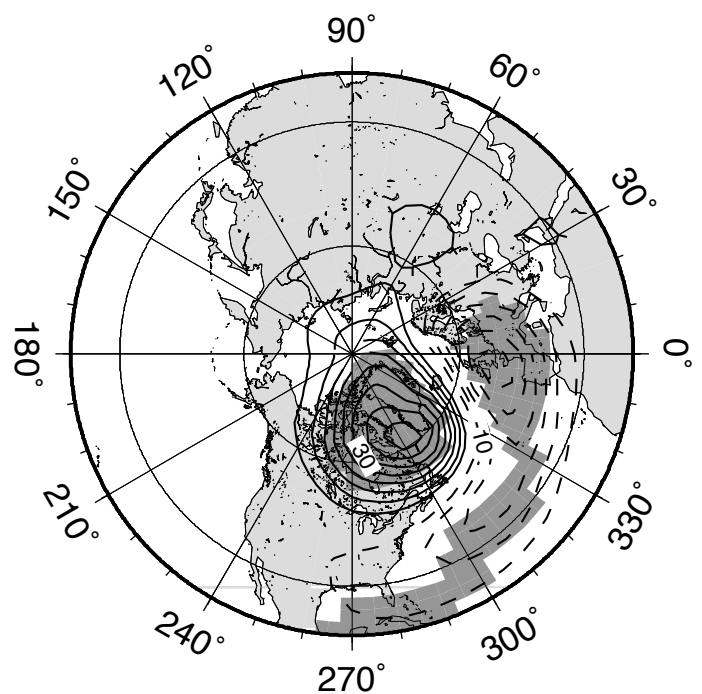

Figure 13. Composites of low-pass filtered 700-hPa geopotential height anomalies for the days assigned into each reanalysis cluster. In each case the 44-winter time average has been subtracted. The shaded regions are significant at the $99 \%$ level according to a two-sided Student $t$-test with 120 degrees of freedom; this number is smaller than the number of days in each composite divided by 10 . Contour interval: $5 \mathrm{~m}$. 


\section{List of Tables}

1 Cluster-wide average measures for various GCM cyclone statistics. Both means

$(\mu)$ and standard deviations $(\sigma)$ are given for each cluster. . . . . . . . . 68

2 Cluster-wide average measures for various reanalysis cyclone statistics. Both means

$(\mu)$ and standard deviations $(\sigma)$ are given for each cluster column. . . . . . . . 69 
TABLE 1. Cluster-wide average measures for various GCM cyclone statistics. Both means $(\mu)$ and standard deviations $(\sigma)$ are given for each cluster.

\begin{tabular}{|l||c|c|c|c|c|c|}
\hline \multicolumn{1}{|l||}{ Cluster-specific statistics } & \multicolumn{2}{c|}{$\mathrm{V}$} & \multicolumn{2}{c|}{$\mathrm{D}$} & \multicolumn{2}{c|}{$\mathrm{H}$} \\
& $\mu$ & $\sigma$ & $\mu$ & $\sigma$ & $\mu$ & $\sigma$ \\
\hline Maximum intensity $(\mathrm{mb})$ & -39.9 & 8.9 & -40.0 & 8.2 & -33.8 & 7.4 \\
\hline Average velocity $(\mathrm{km} / \mathrm{h})$ & 42.5 & 11.5 & 59.4 & 13.8 & 42.8 & 15.9 \\
\hline Average acceleration $\left(\mathrm{km} / \mathrm{h}^{2}\right)$ & 15.4 & 5.7 & 16.5 & 5.6 & 18.8 & 7.5 \\
\hline Lifetime (days) & 3.6 & 0.10 & 4.1 & 1.2 & 3.8 & 1.2 \\
\hline Curvature & 12.1 & 22.0 & 4.8 & 5.2 & 15.2 & 17.8 \\
\hline Noisiness & 19.1 & 48.4 & 7.8 & 13.1 & 23.5 & 35.8 \\
\hline
\end{tabular}


TABLE 2. Cluster-wide average measures for various reanalysis cyclone statistics. Both means $(\mu)$ and standard deviations $(\sigma)$ are given for each cluster column.

\begin{tabular}{|l||c|c|c|c|c|c|}
\hline \multicolumn{1}{|l||}{ Reanalysis cluster-specific statistics } & \multicolumn{2}{c|}{$\mathrm{V}$} & \multicolumn{2}{c|}{$\mathrm{D}$} & \multicolumn{2}{c|}{$\mathrm{H}$} \\
& $\mu$ & $\sigma$ & $\mu$ & $\sigma$ & $\mu$ & $\sigma$ \\
\hline Maximum intensity $(\mathrm{mb})$ & -39.1 & 8.5 & -39.2 & 8.1 & -35.4 & 7.8 \\
\hline Average velocity $(\mathrm{km} / \mathrm{h})$ & 43.9 & 10.5 & 62.6 & 12.9 & 37.8 & 12.76 \\
\hline Average acceleration $\left(\mathrm{km} / \mathrm{h}^{2}\right)$ & 18.0 & 7.1 & 19.6 & 6.9 & 19.5 & 8.0 \\
\hline Lifetime (days) & 3.5 & 0.9 & 3.6 & 1.1 & 3.5 & 1.2 \\
\hline Curvature & 2.9 & 3.1 & 1.8 & 1.9 & 6.2 & 5.5 \\
\hline Noisiness & 4.6 & 6.9 & 3.2 & 3.8 & 8.6 & 9.6 \\
\hline
\end{tabular}

